\title{
Whole-genome assembly and annotation of little yellow croaker (Larimichthys polyactis) provide insights into the evolution of hermaphroditism and gonochorism
}

Qing-Ping Xie ${ }^{1}$, Wei Zhan ${ }^{1}$, Jian-zhi Shi², Feng Liu ${ }^{1}$, Bao-Long Niu ${ }^{1}$, Xue He ${ }^{1}$, Meng Liu ${ }^{2}$, Qiqi Liang ${ }^{2}$, Yue Xie ${ }^{2}$, Peng $\mathrm{Xu}^{3}, \mathrm{Xu} \mathrm{Wang}^{4}$, and Bao Lou ${ }^{1}$

${ }^{1}$ Zhejiang Academy of Agricultural Sciences

${ }^{2}$ Affiliation not available

${ }^{3}$ Xiamen University

${ }^{4}$ Auburn University

August 2, 2021

\begin{abstract}
The evolutionary direction of gonochorism and hermaphroditism is an intriguing mystery to be solved. The special transient hermaphroditic stage makes the little yellow croaker (Larimichthys polyactis, L. polyactis) an appealing model for studying the formation of hermaphrodites. On the other hand, as the most famous commercial fish species in East Asia, the origin and evolutionary relationship of L. polyactis and Larimichthys crocea remain unclear. Here, we report the genome sequence of L. polyactis, with a size of $\sim 706 \mathrm{Mb}$ (contig N50=1.21 Mb and scaffold N50=4.52 Mb) and 25,233 protein-coding genes. Phylogenomic analysis suggests that L. polyactis diverged from the common ancestor of Larimichthys crocea 25.4 million years ago. Our high-quality genome assembly enabled comparative genomic analysis, which revealed a number of within-chromosome rearrangements and translocations without major chromosome fission or fusion events between the two species. The dmrt1 gene was identified as the candidate sex determination gene in L. polyactis. The expression of dmrt1 and its upstream regulatory gene rnf183 were both sexually dimorphic in the transcriptome analysis. Rnf183, unlike its two paralogues rnf223 and rnf225, is only present in Larimichthys but not in other teleost species, suggesting that it originated from a lineage-specific duplication or was lost in other teleosts. Phylogenetic analysis shows that the hermaphrodite stage in male L. polyactis may be explained by the sequence evolution of dmrt1. Decoding the L. polyactis genome not only provides insight into the genetic underpinnings of hermaphrodite evolution but also provides valuable information for enhancing fish aquaculture.
\end{abstract}

Whole-genome assembly and annotation of little yellow croaker (Larimichthys polyactis) provide insights into the evolution of hermaphroditism and gonochorism

Qing-Ping Xie ${ }^{1 \#}$, Wei Zhan ${ }^{1 \#}$, Jian-Zhi Shi6\#, Feng Liu ${ }^{1}$, Bao-Long $\mathrm{Niu}^{1}$, Xue $\mathrm{He}^{1}$, Meng Liu ${ }^{6}$, Qi-Qi Liang $^{6}$, Yue Xie ${ }^{6}$, Peng Xu ${ }^{5 *}$, Xu Wang ${ }^{2,3,4 *}$, Bao Lou ${ }^{1 *}$

1. Institute of Hydrobiology, Zhejiang Academy of Agricultural Sciences, Hangzhou 310021, China;

2. Department of Pathobiology, College of Veterinary Medicine, Auburn University, Auburn, AL 36849, USA;

3. Alabama Agricultural Experiment Station, Auburn, AL 36849, USA;

4. The Hudson Alpha Institute for Biotechnology, Huntsville, Alabama 35806, USA; 
5. State Key Laboratory of Marine Environmental Science, College of Ocean and Earth Sciences, Xiamen University, Xiamen 361005, China;

6. Novogene Bioinformatics Institute, Beijing 100016, China;

\# These authors are Contributed equally to this work

* Corresponding author.

Corresponding authors:

*Peng Xu: State Key Laboratory of Marine Environmental Science, College of Ocean and Earth Sciences, Xiamen University, Xiamen 361005, China;

E-mail addresses:xupeng77@xmu.edu.cn (P. Xu)

*Xu Wang: Department of Pathobiology, College of Veterinary Medicine, Auburn University; Alabama Agricultural Experiment Station; Auburn, AL 36849, USA; The Hudson Alpha Institute for Biotechnology, Huntsville, Alabama 35806, USA;

E-mail addresses:xzw0070@auburn.edu (X. Wang)

*Bao Lou: Institute of Hydrobiology, Zhejiang Academy of Agricultural Sciences, Hangzhou 310021, China;

E-mail addresses: loubao6577@163.com (B. Lou)

Running title: Genome research of little yellow croaker

Word count:7701

Figure number: 8

Keywords: little yellow croaker, whole-genome sequence, gonadal transcriptome, dmrt1 , rnf183 , hermaphrodite evolution

Abstract: The evolutionary direction of gonochorism and hermaphroditism is an intriguing mystery to be solved. The special transient hermaphroditic stage makes the little yellow croaker (Larimichthys polyactis, L. polyactis ) an appealing model for studying the formation of hermaphrodites. On the other hand, as the most famous commercial fish species in East Asia, the origin and evolutionary relationship of L. polyactis and Larimichthys crocea remain unclear. Here, we report the genome sequence of $L$. polyactis, with a size of $\sim 706$ $\mathrm{Mb}$ (contig $\mathrm{N} 50=1.21 \mathrm{Mb}$ and scaffold $\mathrm{N} 50=4.52 \mathrm{Mb}$ ) and 25,233 protein-coding genes. Phylogenomic analysis suggests that L. polyactis diverged from the common ancestor of Larimichthys crocea $\sim 25.4$ million years ago. Our high-quality genome assembly enabled comparative genomic analysis, which revealed a number of within-chromosome rearrangements and translocations without major chromosome fission or fusion events between the two species. The $d m r t 1$ gene was identified as the candidate sex determination gene in $L$. polyactis. The expression of dmrt1 and its upstream regulatory gene rnf183 were both sexually dimorphic in the transcriptome analysis. Rnf183, unlike its two paralogues rnf223 andrnf225, is only present in Larimichthys but not in other teleost species, suggesting that it originated from a lineage-specific duplication or was lost in other teleosts. Phylogenetic analysis shows that the hermaphrodite stage in male L. polyactis may be explained by the sequence evolution of $d m r t 1$. Decoding the $L$. polyactis genome not only provides insight into the genetic underpinnings of hermaphrodite evolution but also provides valuable information for enhancing fish aquaculture.

Key Words: little yellow croaker, whole-genome sequence, gonadal transcriptome, dmrt1 , rnf183 , hermaphrodite evolution

\section{Introduction}

Due to the diversity of ecological niches in the water environment, fishes exhibit a variety of sex determination systems and reproductive patterns. Gonochorism and hermaphroditism are the most important reproductive 
patterns in sexually reproducing organisms and occur commonly in teleost fish (Renner and Ricklefs, 1995; Jarne and Auld, 2006; Bachtrog et al., 2014; Tree of Sex consortium, 2014; Lau et al., 2016); in particular, hermaphroditism has been documented in more than 1,500 species (Sadovy de Mitcheson and Liu, 2008). In fact, males and females are determined by diverse mechanisms that evolve rapidly in fish, yet this diversity in primary sex-determining signals is coupled with conserved molecular pathways that trigger male or female development. The nine taxonomic orders of teleosts in which hermaphroditism has been documented are scattered widely across the phylogeny of teleost fishes, and no order consists solely of hermaphroditic species but instead also contains gonochoristic (separate-sex, or dioecious) species. Interestingly, across all groups, only Perciformes have all types of reproductive patterns and sex differentiation methods, including gonochorism, hermaphrodite protandry, hermaphrodite protogyny, and simultaneous hermaphrodism (Bachtrog et al., 2014; Tree of Sex consortium, 2014; Avise and Mank, 2009). Therefore, whole-genome sequencing of fishes with different reproductive patterns and sexual differentiation methods in Perciformes, especially genome-wide studies of transitional species, will be a powerful tool for the analysis of the evolution of hermaphroditism and gonochorism in fishes.

Little yellow croaker (Larimichthys polyactis, L .polyactis), also named the small yellow croaker or yellow corvina, belongs to the genus Larimichthys, family Sciaenidae and order Perciformes. It is a croaker native to the western Pacific and one of the most famous fish species with great economic importance in China, Korea, and Japan. Little yellow croaker ( $L$. polyactis ) was found to be a good model for investigating the direction of evolution of hermaphroditism and gonochorism due to its special sexual reproduction process, in which males undergo a male-specific transient hermaphroditic stage (Xie et al., 2021). Briefly, L . polyactis is a gonochoristic fish species. When they reach sexual maturity, the sexual functions of males and females are separated, and the sex ratio is close to 1:1. However, in the juvenile stage, EPOs (early primary oocytes) will appear and degenerate between 43 and $80 \mathrm{dph}$ (days post hatching) in the testis, forming an intersex gonad. This unique pattern of testis differentiation is neither typical hermaphroditism nor gonochorism. Because hermaphroditism is extremely rare in the Sciaenidae family, and the closely related species Larimichthys crocea (L. crocea) is a differentiated gonochorist with XX/XY sex determination (You et al., 2012), we speculate that this gonadal differentiation pattern in $L$. polyactis evolved from a gonochoristic ancestor. As the most closely related species in Larimichthys, these species demonstrate two different reproduction systems.

The underlying molecular mechanisms warrant further study. This system may serve as a transitional type from gonochorism to hermaphroditism. The male-specific nature of this transient stage is also fascinating, as it is different from the zebrafish gonadal development process, in which all individuals of zebrafish need to experience the juvenile ovary stage, with no sex differences in morphology and histology (Takahashi, 1977; Orban et al., 2009; Lau et al., 2016). Therefore, whole-genome sequencing of $L$. polyactis is a necessary approach to determine the evolutionary divergence of sexual reproduction patterns at the genome level.

The abundance of wild L. polyactis has severely declined since the 1980 s, and its commercial value has increased significantly due to overfishing, seawater pollution, and ocean current and water quality changes (Zhang et al., 2016; Han et al. 2010). Although the resources of $L$. polyactis have gradually recovered (Cheng et al. 2004), overfishing has caused sexual precocity, gender imbalance, and growing miniaturization in $L$. polyactis populations (Jin 1996; Lin and Cheng 2004). Therefore, artificial breeding techniques are urgently needed to restore fishery resources. In 2015, an artificial breeding programme was successfully established by artificial spawning of wild $L$. polyactis and larval culture (Chen et al. 2016; Liu et al., 2019; Xie et al., 2021). A previous report of a genetic linkage map and karyotype analysis revealed that $L$. polyactis is a diploid $(2 \mathrm{n}=48)$ (Liu et al., 2020; Park and Gil, 2019). A high-quality assembled genome of L . polyactis will provide the necessary toolkit for studies of genome structure and evolution in its reproductive patterns and sex differentiation methods. In this study, to elucidate the role of genomic architecture in hermaphrodite evolution, we produced a high-quality reference genome $(706 \mathrm{Mb})$ of $L$.polyactis . Transcriptome data were also obtained from 13 major organs and 18 gonadal development time points from both sexes. We have established an excellent model for studying the relationship between hermaphroditism and gonochorism in teleosts, and the discovery of a transient juvenile intersex stage in males also enriches the theory of teleost 
sex determination and differentiation.

\section{Materials and Methods}

\section{Samples and sequencing}

The little yellow croaker L. polyactis (National Center for Biotechnology Information [NCBI] Taxonomy ID: 334908; Fishbase ID: 1877) female and male individuals (Figure 1A) were collected from an artificially bred F2 generation (2016) in Zhoushan, Zhejiang Province, China. The $L$. polyactis genome file has been submitted to NCBI (National Center for Biotechnology Information,https://www.ncbi.nlm.nih.gov), and the accession number is JAFMOB000000000. High-quality genomic DNA was extracted from the muscle and blood of a male fish using a Qiagen GenomicTip100 (Qiagen, Hilden, USA) for sequencing on the PacBio platform. To build a 20-kb insert size library, the first step was template preparation, which involved DNA concentration, damage repair, end repair, ligation of hairpin adapters, and template purification using AMPure PB Magnetic Beads. Then, the sequencing primer was annealed, and sequencing polymerase was bound to the SMRTbell template. Finally, the library was sequenced using the Pacific Biosciences RSII instrument, yielding approximately $48 \mathrm{~Gb}$ PacBio data ( $~ 69$-fold coverage of the genome). At the same time, we constructed a short-insert (350 bp) paired-end library using a sample preparation kit following the manufacturer's recommendations, and the library was sequenced on the Illumina HiSeq platform with a 150 bp read length, obtaining approximately $128 \mathrm{~Gb}$ of data ( 183 -fold coverage of the genome). In addition, other mapping technologies, including BioNano genome mapping and 10× Genomics linked reads, were also used to generate a high-quality reference sequence of $L$. polyactis. A total of $\sim 431 \mathrm{~Gb}$ of sequencing data was obtained ( $~ 616$-fold coverage of the genome). Animal experiments were conducted in accordance with the regulations of the Guide for Care and Use of Laboratory Animals, which was approved by the Committee of Laboratory Animal Experimentation at Zhejiang Academy of Agricultural Sciences.

\section{Estimation of genome size}

To estimate the genome size of $L$. polyactis, we used reads from paired-end libraries to determine the distribution of K-mer values. The distribution of $\mathrm{K}$-mers depends on the characteristics of the genome and follows Poisson's distribution ( $\mathrm{Li}$ et al., 2010). A total of $128 \mathrm{~Gb}$ of high-quality short-insert reads (350 bp) were used to estimate the $L$. polyactis genome size based on the 17-mer frequency distribution using the following formula: Genome size $=($ total number of 17-mers) $/($ position of peak depth) $($ Liu et al., 2013).

\section{Genome assembly}

\section{PacBio long-read de novo assembly}

First, the PacBio reads were subjected to error correction according to the rate of insertions, deletions, and sequencing errors between the base pairs to obtain preassembled reads using daligner (Myers, 2014). Then, the preassembled reads were assembled by the consensus algorithm called Overlap-Layout-Consensus (DBG2OLC) to obtain contigs using FALCON (v1.2.4) (Pendleton et al., 2015). Finally, the assembled contigs were subjected to error correction with the PacBio data by Quiver (v5.0.1) (Chin et al., 2013), and the short-insert size (350 bp) reads were corrected by pilon (v1.22) (Walker et al., 2014).

\section{Scaffold assembly}

To construct superscaffolds, the PacBio contigs were first scaffolded using fragScaff (Adey et al., 2014) according to the location and distance information among linked reads produced from the 10x Genomics libraries, and then, the resulting scaffolds were connected to superscaffolds using optical map data. A highdensity genetic map was built by chromonomer (version 1.07) (Catchen et al, 2020) to anchor the scaffolds into a chromosome level genome.

\section{Genome evaluation}

\section{BWA evaluation}


To evaluate the quality of the genome assembly, we mapped the short-insert size reads back to the scaffolds using BWA ( $\mathrm{Li}$ et al., 2009) with the following parameters: "-o 1 -i 15". The sequencing depth distribution follows a Poisson distribution, which indicates the uniformity of the genome sequencing data.

\section{CEGMA and BUSCO evaluation}

CEGMA (Core Eukaryotic Genes Mapping Approach) (Parra et al., 2007) uses a highly reliable set of 248 conserved protein families that occur in a wide range of eukaryotes to evaluate genome assembly. It has a series of procedures that can accurately identify the exon-intron structures of conserved genes in a novel genomic sequence. BUSCO (Benchmarking Universal Single-Copy Orthologs) (Simão et al., 2015) was combined with TBLASTN (Altschul et al., 1997), Augustus (Stanke and Waack, 2003; Burkhard et al., 2006) and HMMER (Mistry et al., 2013) to assess the completeness of the genome assembly.

\section{Genome annotation}

\section{Repeat detection}

After genome assembly, repeat annotation was carried out for theL. polyactis genome. We identified transposable elements (TEs) with a combination of homology and de novo prediction methods using multiple software packages. First, we built the de novorepeat library using RepeatModeler (Smit et al.), RepeatScout (Price et al., 2005), and LTR_FINDER (Xu et al., 2007). Second, we performed homology-based prediction using RepeatMasker with default parameters against Repbase and de novo repeat libraries at the DNA level. In addition, we used RepeatProteinMask (Tarailo-Graovac and Chen, 2009) with the default parameters to identify repeat sequences at the protein level. The tandem repeats were searched using Tandem Repeats Finder (Benson, 1999) with the parameters "Match=2, Mismatch=7, Delta=7, PM=80, PI=10, Minscore=50, and MaxPeriod=2000".

\section{Protein-coding gene annotation}

We predicted the protein-coding genes with a combination of homology-based gene prediction, de novo methods, and RNA-seq data, and then, all predicted genes were integrated by EVidenceModeler (Haas et al., 2008) to obtain nonredundant data.

For homology-based gene prediction, we used the longest protein-coding sequences of the genes from eleven species, including L. crocea, Oreochromis niloticus, Gasterosteus aculeatus, Oryzias latipes, Takifugu rubripes, Cynoglossus semilaevis, Cyprinus carpio, Danio rerio, Homo sapiens, Mus musculus and Branchiostoma floridae, for analysis (Wang et al., 2014). First, we aligned those protein sequences against our assembled genome with an E-value $<1 \mathrm{e}-5$ using TBLASTN (Altschul et al., 1997) and linked the alignment results with SOLAR (Darriba et al., 2012). Then, Genewise (version 2.2.0) (Birney et al., 2004) was used to predict gene models based on the alignment sequences. The homology-based gene structures were obtained.

We used Augustus (version 3.0.2) (Stanke \& Waack, 2003; Stanke et al., 2006), Genescan (version 1.0) (Salamov and Solovyev, 2000), Geneid (version 1.4) (Parra et al., 2000), GlimmerHMM (version 3.0.2) (Majoros et al., 2004) and SNAP (Ian, 2004) for de novo gene prediction on the repeat-masked genome. Finally, we obtained five results from the five methods.

In addition, RNA-seq data from different tissues were used to assist in gene prediction. On the one hand, we mapped the RNA-seq data to the L. polyactis genome using TopHat (version 2.0.13) (Trapnell et al., 2009) and obtained gene structures by cufflinks (version 2.1.1) (Trapnell et al., 2010) based on alignment positions. On the other hand, we assembled the RNA-seq data by Trinity (version 2.1.1) (Grabherr et al., 2011) and then obtained the gene structures by PASA (Hass et al., 2003) software. We merged all gene structures obtained by the three approaches using EVidenceModeler (Haas et al., 2008) to generate the nonredundant gene sets. Finally, we used PASA (Hass et al., 2003) to acquire the final gene structures after adjusting the gene models generated from EVidenceModeler with the transcripts assembled by Trinity (Grabherr et al., 2011).

\section{Functional annotation}


We carried out gene function annotation to better understand the biological adaptation of the species. First, we used our coding proteins to search against InterPro (Mulder et al., 2007) databases, including Pfam (Finn et al., 2000), PRINTS (Attwood and Beck, 1994), PROSITE (Hulo et al., 2006), ProDom (Bru et al., 1998), SMART (Letunic et al., 2012) and PANTHER (Mi et al., 2005), by InterProScan (version 4.7) (Mulder et al., 2007) to obtain motifs and domains. Moreover, we obtained the Gene Ontology (GO) (Ashburner, 2000) terms for each gene from the corresponding InterPro35 descriptions. We also searched KEGG (Kyoto Encyclopedia of Genes and Genomes) (Hiroyuki et al., 2000) databases to determine which pathway the gene might be involved in, as well as SwissProt and TrEMBL (Bairoch and Apweiler, 2000) databases to find the gene symbols.

\section{Noncoding RNA annotation}

Noncoding RNA (ncRNA) is a kind of RNA molecule that cannot be translated into a protein. Here, we annotated four types of ncRNAs, including microRNAs (miRNAs), transfer RNAs (tRNAs), ribosomal RNAs (rRNAs), and small nuclear RNAs (snRNAs). We searched for rRNAs by blasting the rRNA database and searched for siRNAs and snRNAs against the Rfam (Griffithsjones et al., 2005) database by INFERNAL (Nawrocki et al., 2009) software. Finally, we used tRNAscan-SE (Lowe and Eddy, 1997) to search for tRNAs in the genomes.

\section{Genome comparison}

\section{Gene family cluster}

Orthologues are homologous sequences resulting from a single ancestral sequence in the last common ancestor of an examined species by a speciation event (Yang et al., 2016). We carried out gene family clustering using OrthoMCL (Li et al., 2016) with 18 species, L. polyactis, L. crocea, Oreochromis niloticus , Gasterosteus aculeatus, Oryzias latipes, Takifugu rubripes, Cynoglossus semilaevis, Danio rerio , Homo sapiens , Mus musculus, Gallus gallus , Branchiostoma floridae, Lepisosteus oculatus, Callorhinchus milii, Gadus morhua , Ciona intestinalis, Anolis carolinensis and Xenopus tropicalis . First, we obtained the longest transcript of the genes and removed the genes whose protein length was less than 30 amino acids. Then, we found possible matches using the 18 species' protein sequences by running an all-vs-all blast with an E-value cutoff of 1e-7. Finally, we clustered the alignments into gene families using OrthoMCL (Li et al., 2016).

\section{Phylogenetic analysis at the gene and genome levels}

Phylogenetic relationships at the genome level were analysed for eighteen species, L. polyactis, L. crocea ,Oreochromis niloticus, Gasterosteus aculeatus ,Oryzias latipes, Takifugu rubripes, Cynoglossus semilaevis , Danio rerio, Homo sapiens, Mus musculus , Gallus gallus , Branchiostoma floridae ,Lepisosteus oculatus , Callorhinchus milii, Gadus morhua, Ciona intestinalis , Anolis carolinensis, andXenopus tropicalis . First, the amino acid sequences from the 590 single-copy genes among the 18 species were aligned individually by MUSCLE (Edgar and Robert, 2004). Then, we concatenated one supergene with the single-copy gene alignments and constructed a phylogenetic tree using the maximum likelihood (ML) method in RAxML software (version 8.0.19) (Stamatakis, 2006; Stamatakis et al., 2008). The phylogenetic relationships of RING-finger protein ( $r n f$ ) family members on chromosome 2, rnf183, rnf223, and rnf225, and dmrt1 were analysed among the following species: Homo sapiens, Mus musculus, Alligator sinensis, Chelonia mydas ,Xenopus tropicalis, Xenopus laevis, Xenopus tropicalis, Gallus gallus, Trachemys scripta, Danio rerio , Takifugu rubripes, L. crocea, L. polyactis ,Oreochromis niloticus, Lates calcarifer, Amphiprion ocellaris , Oryzias latipes, Kryptolebias marmoratus, Acanthopagrus schlegelii, Labrus bergylta ,Epinephelus merra , Epinephelus coioides, Epinephelus lanceolatus, Centropristis striata, Monopterus albus ,Sparus aurata, Oncorhynchus mykiss, Gadus morhua, and Anolis carolinensis. The GenBank accession number of each gene and species is listed in Supplementary Table S1. The evolutionary history was inferred by using the maximum likelihood method and general time reversible model (Nei and Kumar, 2000). The tree with the highest log-likelihood is shown. The percentage of trees in which the associated taxa clustered together is shown next to the branches. Initial tree(s) for the heuristic search were obtained automatically by applying neighbour-joining and BioNJ algorithms to a matrix of pairwise distances estimated using the maximum 
composite likelihood (MCL) approach and then selecting the topology with a superior log-likelihood value. A discrete gamma distribution was used to model evolutionary rate differences among sites. The rate variation model allowed some sites to be evolutionarily invariable. The tree is drawn to scale, with branch lengths measured as the number of substitutions per site. This analysis involved several nucleotide sequences. Evolutionary analyses were conducted in MEGA X (Kumar et al., 2018; Stecher et al., 2020). According to the results of the phylogenetic tree, we corrected the nomenclature of rnf225 and rnf183 in the following species: rnf225 has been wrongly named rnf183 in Danio rerio, Oryzias latipes, Kryptolebias marmoratus , Oreochromis niloticus ,Amphiprion ocellaris, and Epinephelus lanceolatus .

\section{Divergence time estimation}

Divergence time estimation was implemented based on the phylogenetic tree and CDSs by the mcmctree program of PAML (Yang, 2007) (http://abacus.gene.ucl.ac.uk/software/paml.html) among 18 species with the main parameters "burn-in $=10000$, sample-number $=100,000$ and sample-frequency $=2 "$. Seven calibrating time points were selected from the TimeTree (Hedges et al., 2015) website (http://www.timetree.org):Homo sapiens and Mus musculus , 61 100 million years ago (Mya); Gallus gallus and Anolis carolinensis, 225 239 Mya; Homo sapiens and Gallus gallus , 312 230 Mya; Gasterosteus aculeatus, L. crocea, L. polyactis, and Takifugu rubripes, 97 151 Mya; Gadus morhua ,Cynoglossus semilaevis, Gasterosteus aculeatus, L. crocea , L. polyactis , Takifugu rubripes ,Oreochromis niloticus, and Oryzias latipes, 139 158 Mya; Danio rerio and nine other species, 149 165 Mya; and Callorhinchus milii and fifteen other species, $422^{\sim} 463$ Mya.

\section{Gene family contraction and expansion}

We explored gene families that underwent a significant change among $L$. polyactis and other species based on the abovementioned phylogenetic tree and divergence time. We performed expansion and contraction analysis using the CAFÉ (Version 2.1) (De et al., 2006) program after filtering the families that had more than 200 copies in one species had less than 2 copies in other species. By comparing each branch with their ancestor branch, the $P$-value was calculated using Fisher's exact test and was then adjusted by the false discovery rate (FDR). Families with an adjusted p-value less than 0.05 were considered to have a significant change, which means they underwent contraction or expansion during the process of evolution (Jeffery et al., 2006). The L. polyactis genes included in the contracted or expanded families were extracted and subjected to GO/KEGG enrichment analyses.

\section{Genes with accelerated evolutionary rates}

Positive selection analysis was carried out at the DNA sequence level by estimating the ratio $(\omega)$ of nonsynonymous nucleotide substitutions $(\mathrm{dN})$ to synonymous nucleotide substitutions (dS) between orthologous genes used in the phylogenetic analysis (Sun et al., 2012). We calculated different $\omega$ values in the phylogenetic tree, including $\omega 0, \omega 2$, and $\omega 1$, representing the whole tree, foreground branch, and background branch, respectively, with the branch-site model (Zhang et al., 2005; Zhang et al., 2013). P-values were computed using the $\chi 2$ statistic and adjusted by the FDR method. Genes with an adjusted p-value less than 0.05 were thought to have undergone positive selection. We ran three groups of positive selection analyses. In the first group, we set L. polyactis as the foreground branch and L. crocea as the background branch, and 955 positively selected genes were found in the $L$. polyactis genome. In the second group, we set L. polyactis as the foreground branch and Oryzias latipes ,Oreochromis niloticus, Takifugu rubripes, L. crocea, and Gasterosteus aculeatus as the background branch, and 761 positively selected genes were found in the $L$. polyactisgenome. In the third group, we set L. polyactis as the foreground branch and Takifugu rubripes, $L$. crocea, and Gasterosteus aculeatus as the background branch, and 1080 positively selected genes were found in the L. polyactis genome.

\section{Comparison of the L. polyactis and L.crocea genomes}

Similar to $L$. crocea , $L$. polyactis has 24 chromosomes. To visualize the concordance with the $L$. croceagenome, the 24 chromosomes were aligned to $L$. crocea using blastp (blastp -e 1e-10 -b 5 -v 5 m 8) (BLASTP, RRID:SCR_001010). Gene alignments of both nucleic acid and amino acid sequences 
were concatenated and submitted to RAxML for maximum likelihood (ML) inference. The amino acid phylogeny was inferred using the GAMMA AUTO model, which enables RAxML to automatically determine the best-scoring protein substitution model (Stamatakis, 2014). The L. croceagenome was obtained using GenBank: CM011695.1 from NCBI (https://www.ncbi.nlm.nih.gov/nuccore/1523709032/). The results were plotted using circos (version 0.69) (Krzywinski et al., 2009) and mcscan (https://github.com/tanghaibao/jcvi/wiki/MCscan-(Python-version) (Tang et al., 2008). The synteny inference of sex chromosomes was constructed by using JCVI utility libraries (Tang et al., 2008), which is a python package. The alignment of the two genomes was carried out by using the MUMMER alignment algorithm (MUMMER).

\section{Gonadal RNA sequencing}

Total RNA was extracted from thirteen kinds of tissues, including gill, muscle, kidney, head kidney, liver, spleen, brain, eye, heart, skin, testis, ovary, and intestine, and genomic DNA contamination was removed. The integrity of the RNA was evaluated on an agarose gel stained with ethidium bromide (EB), and its quality and quantity were assessed using a NanoDrop and Agilent 2100 Bioanalyzer. Then, cDNA libraries were constructed following the manufacturer's recommendations and sequenced on the Illumina HiSeq platform, generating 150-bp paired-end reads. A summary of the clean data from different tissues is shown in Supplementary Table S2. These RNAseq libraries were also sequenced to assemble transcriptomes and annotate genes.

Total RNA was extracted from three mixed pools of whole-fish samples at 7, 10, 20, and $30 \mathrm{dph}$, where each pool had six fishes; from three mixed pools of indistinguishable gonads at $40 \mathrm{dph}$, where each pool had six gonads; and from thirteen pairs of ovary and testis mixed pools at 50, 60, 70, 80, 100, 130, 180, 240, 270, 340, 360, 400, and $720 \mathrm{dph}$, where each pool had three samples. The genomic DNA contamination was removed. The integrity of the RNA was evaluated on an agarose gel stained with ethidium bromide (EB), and its quality and quantity were assessed using a NanoDrop and Agilent 2100 Bioanalyzer. Then, cDNA libraries were constructed following the manufacturer's recommendations and sequenced on the Illumina HiSeq platform, generating 150-bp paired-end reads. High-quality reads from each sample were mapped onto the assembled transcript sequences using Bowtie software (Langmead and Salzberg, 2012) with default parameters. The RSEM program (Li and Dewey, 2011) was then used to estimate the expression abundance of the transcripts. The total number of mapped reads for each transcript was determined and then normalized to determine RPKM (reads per kilobase of transcript per million mapped reads).

\section{Statistical Analysis}

Statistical software uses SPSS statistics v17.0 (Chicago, USA). Independent sample t-test, one-way ANOVA, and Duncan's post hoc test were performed to determine the statistical significance at $\mathrm{P}<0.05$.

\section{Results}

\section{Genome sequencing assembly and annotation}

We sequenced the L. polyactis (Figure 1A) genome using a combination of different approaches, including single-molecule real-time (SMRT) sequencing technology, Illumina short-insert size (350 bp) sequencing technology, and new mapping technologies (BioNano genome map and 10× Genomics linked reads). A total of $\sim 431 \mathrm{~Gb}$ of data was obtained ( $\sim 616$-fold coverage of the genome) (Table 1$)$. A total of 715 scaffolds were assembled, covering a total length of the final assembly of $\sim 706 \mathrm{Mb}$. The calculated contig N50 and scaffold N50 were $1.21 \mathrm{Mb}$ and $4.52 \mathrm{Mb}$, respectively (Table 2). The G-C content of the assembly was $41.45 \%$ (Supplementary Table S3, S4). Based on the constructed genetic map, scaffolds were mapped to 24 chromosomes, accounting for $88.55 \%$ of the total length (Table 2).

To evaluate the consistency and completeness of the genome assembly, we performed short-insert read mapping by BWA. The mapping rate was $97.49 \%$, covering $99.36 \%$ of the genome with a homologous SNP rate of $0.03 \%$. To assess the completeness of the genome assembly (Supplementary Table S5), 248 ultraconserved 
eukaryotic genes were examined by CEGMA9, and 93.95\% were found in our assembly, indicating high integrity of the core genes (Supplementary Table S6). BUSCO analysis was also conducted, a total of 4,304 out of the 4,584 searched BUSCO groups (93.9\%) had been completely assembled in our genome, suggesting a high level of completeness of the de novo assembly (Supplementary Table S7). A total of $157 \mathrm{Mb}(\sim 22 \%)$ of genome sequences was predicted to be repetitive elements, including $38 \mathrm{Mb}$ (5.34\%) of long-terminal repeat (LTR) retrotransposons and $67 \mathrm{Mb}(9.57 \%)$ of DNA transposons (Supplementary Table S8-S9).

For the chromosome-level assembly, a high-density linkage map was used to anchor the scaffolds. A total of 309 scaffolds were anchored to 24 pseudochromosomes, representing $88.55 \%$ of the total genome (Table 1).

A total of 25,233 protein-coding genes were predicted using a standard annotation pipeline, and $84.3 \%$ of the genes were supported by homologous protein sequences or transcriptomic data (Table 1; Figure 1B; Supplementary Table S10-S11). The sequence divergence rate for each family of TEs was calculated (Figure $1 \mathrm{C})$.

Gene family analysis of $L$. crocea, Gasterosteus aculeatus, Danio rerio, and L. polyactis identified a core set of 10,215 gene families, 26,313 gene families, and 590 single-copy gene families among L. polyactis and seventeen other species; we counted and plotted the number of orthologous genes across the eighteen species in a Venn diagram (Figure 1D-E). On average, the genes had an exon length of $166.59 \mathrm{bp}$ and intron length of 1,270.49 bp (Supplementary Table S10). Approximately 95.5\% (24086) of the predicted genes were assigned functional annotations by alignment against various databases, including InterPro35, KEGG43, SwissProt45, and TrEMBL45 (Supplementary Table S12). Four types of noncoding RNAs (ncRNAs), including microRNAs (miRNAs), transfer RNAs (tRNAs), ribosomal RNAs (rRNAs), and small nuclear RNAs (snRNAs), are annotated in Supplementary Table S13.

\section{Genome evolution analysis}

Phylogenetic analysis based on 590 selected 1:1 orthologous genes suggests that the L. polyactis lineage diverged from the common ancestor of the $L$. crocea lineage $\sim 25.4$ million years ago. The ancestor of $L$. polyactis and $L$.crocea separated from the ancestor of $T$. fuguapproximately 90.1 million years ago. Genefamily analysis among the 18 species found 320 expanded gene families and 544 contracted families on the $L$. polyactis branch (Figure 2). The three groups of positive selection analyses found that more than 700 genes underwent accelerated evolution. The L. polyactis genes included in the contracted or expanded families were extracted and subjected to GO/KEGG enrichment analyses. The expanded gene families and the positively selected genes were all probably important for L. polyactis adaptations (Figure 2, Supplementary Tables S14-S23).

\section{Comparative genomic analysis of the sex chromosomes}

Comparison of the genomes of $L$. polyactis and $L$.crocea revealed that 24 chromosomes of $L$. polyactiscould be unambiguously aligned to single chromosomes of $L$.crocea (Figure 3 ). The results reflected the high quality of the $L$. polyactis genome assembly. Although these chromosomes did not experience chromosome fission and fusion events, a number of rearrangements and translocations were found in pairwise comparisons (Figure 3).

Syntenic analysis of the sex chromosomes of L. crocea (linkage group 22, LG22) and L. polyactis (chromosome 2, Chr. 2) revealed that one scaffold (scaffold 8) was inserted into Chr. 2 of L. polyactis and that the other sequences had high collinearity. Interestingly, six members of the ring finger protein ( $r n f$ ) family and several genes involved in sex differentiation and gonadal development, including the sex determination candidate genedmrt1, were identified in the sex determination region (Figure 4A). A $4.62 \mathrm{Mb}$ region surrounding dmrt1 (from piwi2 tornf122) in L. polyactis was compared to the corresponding syntenic region in L. crocea (4.73 $\mathrm{Mb})$. Duplication of a protein-coding gene in the cilia- and flagella-associated protein family, cfap157, was also found in L. polyactis (Figure 4B). Since a large number of rnf genes were not observed in any other fish clade, we analysed the phylogenetic relationship of these sex-linked rnf genes and autosomal members inLarimichthys . The results showed that rnf family members have a high degree of homology between these 
two species, L. polyactis and L. crocea (Figure 4C).

\section{Identification and expression analysis of the sex-determining candidate gene dmrt1}

Transcriptome analysis was performed on thirteen L. polyactistissues, gill, muscle, kidney, head-kidney, liver, spleen, brain, eye, heart, skin, testis, ovary, and intestine. Gonad gene expression profiling was also conducted at 18 developmental stages in whole-fish samples at 7, 10, 20, and $30 \mathrm{dph}$, indistinguishable sex gonads at 40 $\mathrm{dph}$, and testes and ovaries at 13 later stages (see Methods). The early-stage gonads before $40 \mathrm{dph}$ clustered together; indistinguishable sex gonads at $40 \mathrm{dph}$ and testes before meiosis (50-180, 400 and $720 \mathrm{dph}$ ) clustered together; and initiation of meiosis to form the functional testis of sexual maturity (240-360 dph) clustered together. From $50 \mathrm{dph}$, all stages of ovarian development clustered together (50-720 dph). A panel of sex determination- and differentiation-related genes, includingcyp19a1a, figla, rnf183, foxl2, rnf225, gsdf, amh, and dmrt1, were annotated in the genome and transcriptome data (Figure 5 and Supplementary Figure S1). Among them, cyp19a1a, figla, rnf183, and foxl2 showed an ovary expression bias, whilernf225, gsdf, amh, and dmrt1 showed a significant testis bias. If we restricted the genes in the sex determination region,dmrt1 was testis-specific in all stages (Figure 6), andrnf183 (Figure 7) and figla (Figure 8) were significantly ovary biased. Therefore, we conclude that $d m r t 1$ is the best sex determination gene candidate.

A phylogenetic tree was constructed from $d m r t 1$ nucleotide sequences. The results showed that most of the fish species analysed could be clustered in one main group (Figure 6A). Most of them are Perciformes, except for Oryzias latipes, Kryptolebias marmoratus, and Monopterus albus . Similarly, the closest relative of $L$. polyactis based on dmrt1 is still $L$. crocea . The fish group contains gonochorist, juvenile hermaphrodite, hermaphrodite protandry and hermaphrodite protogyny. Interestingly, the branches of L. polyactis and $L$. croceawere located between species with hermaphrodite protandry and hermaphrodite protogyny, including Epinephelus merra, Epinephelus coioide ,Labrus bergylta, Centropristis striata ,Acanthopagrus schlegeli, Lates calcarifer andMonopterus albus. Oreochromis niloticus, Oryzias latipes and Takifugu rubripes are typical gonochorists, and they are scattered in different branches. Danio rerio belongs to the juvenile hermaphrodite group and is distributed in a separate branch. The other main group includes mammals, reptiles, birds, and amphibians, which are gonochorists. The reproductive patterns of these species are shown in the diagram (Figure 6A). These results indicated thatdmrt1 was correlated with the evolution of gonochorism and hermaphroditism.

A gene locus map showed that the gene orientation of $d m r t 1$ and its downstream genes $d m r t 3, d m r t 2$, and smarca2 is conserved among mammals, reptiles, amphibians, and other teleosts, except Larimichthys . The gene order was rearranged, with anrnf183 gene insertion immediately upstream of dmrt1 inLarimichthys . Between the closely related T. rupries andLarimichthys, the upstream gene contents were different, suggesting rapid genomic evolution in this region (Figure 6B). The results indicated that rnf183 is present only inLarimichthys and not in any other teleost species.

The gene structure of dmrt1 contains 5 exons, resembling that of other teleost species, including $D$. rerio , O. latipe, T. rubripes, O. niloticus and L. crocea. Compared with that of its close relative L. crocea , the fourth exon has an 18-bp insertion (Figure 6C). Further analysis of the transcription profiles in its gene neighbourhood identified an immediate upstream gene, rnf183, whose expression was also sexually dimorphic. Analysis of the transcripts of rnf183 revealed that this gene has two exons and is only 3197 bases away from dmrt1 (Figure 6D).

\section{Rnf183 and its close paralogues show a pattern of rapid evolution in teleosts}

Rnf183is $3 \mathrm{~kb}$ upstream of dmrt1, and it displayed an opposite sex-biased pattern in gonad expression compared to $d m r t 1$. Its ovary-specific expression may result in testis-specific expression of $d m r t 1$ through a collateral damage effect. We investigated the evolutionary history of rnf183 and found that two autosomal genes, rnf223 and rnf225, are closely related paralogues. Orthologues for all three genes could be found in mammals, reptiles, and amphibians. Although rnf223 and rnf225 are present in most fish species we examined, rnf183 can only be found in Sciaenidae, Latidae, Pomacentridae, Labridae, Sparidae, Salmonidae, and Cichlidae, suggesting gene loss during evolution (Figure 7A). 
A gene locus map showed that the gene orientation of rnf183 and its upstream and downstream genes is relatively conserved among mammals, amphibians, and reptiles but not among teleosts. rnf183 is next to rnf223 in Xenopus, suggesting that they may have been derived from an ancient duplication event. The sex-linked location of 19183 is only found in Larimichthys, and its gene neighbourhood is very different from that of other fish species withrnf183 orthologues (Figure 7B).

The tissue distribution and gonadal expression of rnf183 ,rnf223 (on Chr. 11), and rnf225 (Chr. 24) were analysed,rnf183 was expressed at low levels in gills/testes and was extremely highly expressed in ovaries (RPKM >120).rnf225 was highly expressed in gills (RPKM > 120), with only a very low level of expression detected in skin and other tissues. rnf223 was expressed broadly in multiple tissues, with a moderate level in the testis (RPKM > 10) but no expression in the ovary (Figure 7C). These results indicated the functional divergence of rnf183, rnf223, and rnf225, presumably due to subfunctionalization after gene duplication. Whilernf223 and rnf225 maintain their expression and function in the gill and other tissues, rnf183 specializes in sex determination, differentiation or maintenance in the ovary of L. polyactis .

\section{Sex-related gene expression profiles in L. polyactis}

A few well-identified and studied sex-related genes were screened to analyse expression profiles. First, Amh (anti-Müllerian hormone, on Chr. 6) and gsdf (gonadal soma-derived factor, on Chr. 16) are gonad-specific and highly expressed in the testis. Amh initiated its expression from $40 \mathrm{dph}$ before testicular meiosis (50-180 $\mathrm{dph}$ ), and it was downregulated during spermatogenesis to mature testis. In contrast to amh, Gsdf was expressed as early as $7 \mathrm{dph}$ and maintained high expression during the juvenile (7-40 dph) and later stages (after $50 \mathrm{dph}$ ). However, high expression of gsdf was also detected in the ovary when entering the breeding season (360 and $720 \mathrm{dph}$ ). Cyp19a1a (cytochrome P450, family 19, subfamily A, polypeptide 1a, on Chr. 10), figla (factor in germline alpha, on Chr. 2) and foxl2 (Forkhead Box L2, in Chr. 15) are considered tightly related to oestrogen synthesis, oocyte formation, and ovarian differentiation. Although cyp 19a1a was widely expressed in many tissues and expressed as early as $7 \mathrm{dph}$, in some cases, its expression was significantly higher in ovaries than in testes. Figla is mainly expressed in the gonads, especially in the ovary. It was first detected at $40 \mathrm{dph}$ and maintained a significantly higher level in the ovary than in the testis. Foxl2 was mainly expressed in the gill, eye, brain, and gonad, whereas it was more highly expressed in the ovary than in the testis at a few stages. Interestingly, these three genes were all expressed in the testis in the transient hermaphrodite stage (43-80 dph) in L. polyactis, and even at this stage, there were no significant differences in the expression levels of cyp19a1aand foxl2 in the testis and ovary, except foxl2 at 50 dph (Figure 8). These results indicated that the transient hermaphrodite stage (43-80 dph) in L. polyactis has a complex formation basis and molecular mechanism.

\section{Discussion}

\section{A high-quality genome assembly of $L$. polyactis}

L. polyactis is a commercially important Sciaenidae fish species. Adult fish show sexual dimorphism, with females remarkably larger than males. A reference genome is crucial for biological studies of L. polyactis . In the present study, we assembled the most contiguous genome of L. polyactis via the integration of nanopore long-read sequencing, Bionano, and 10x Genomics technologies. This is the first high-quality de novo assembled genome in Sciaenidae using third-generation sequencing.

Our assembly benefited from long-read sequencing technologies. The combination of long-read assembly and Illumina short-read correction ensured the accuracy and completeness of the genome assembly. The contig N50 is much longer with a smaller number of contigs than the genome assembly of several fish species using third-generation sequencing (Table S17) (Gong et al., 2018; Cai et al., 2019; Conte et al., 2019; Ge et al., 2019; He et al., 2019; Jiang et al., 2019; Yang et al., 2019). Physical mapping using Bionano and 10x Genomics linked reads further improved the quality of the genome assembly to the pseudochromosome level. rnf183 and its paralogues can be identified and annotated, which provides another indication of the high quality of gene assembly. 
A total of 25,233 protein-coding genes were annotated in the L. polyactis genome, 23,991 of which had functional annotations. A total of 5,671 non-coding RNAs were also identified. In addition, the genome contained $22.28 \%$ repetitive sequences, which was lower than the genomes of Danio rario (52.5\%), Epinephelus lanceolatus(41.01\%), Epinephelus. akaara (43.02\%), Glyptosternon maculatum (33.96\%), and Gasterosteus aculeatus (25.2\%).

Most teleosts have undergone teleost-specific genome duplication (TGD), and their haploid genomes contain 24 to 25 chromosomes. The number of chromosomes assembled in the genome of L. polyactis was 24 , which was consistent with the L. crocea and karyotype analysis (Liu et al., 2020; Park and Gil, 2019). By compared the genomes of these close species, although these chromosomes did not experience chromosome fission and fusion events, a number of rearrangements and translocations were found in pairwise comparisons.

\section{A model for studying the evolution of hermaphroditism and gonochorism in fish}

Theoretically, gonochorism and simultaneous hermaphroditism are the two evolutionary endpoints on a reproductive spectrum (Avise and Mank, 2009), where gonochorism could evolve from hermaphroditism either by gradually increasing sex-specific investment or the occurrence of male- or female-sterility mutations (Bachtrog et al., 2014). The opposite also exists; hermaphroditism could evolve from gonochorism due to sexual reproduction, and sex determination genes or loci have been lost and re-evolved many times in plants (Schaefer H, Renner SS, 2010).

In fish, the direction of evolution between hermaphroditism and gonochorism remains unclear due to the lack of transitional species between the above two reproductive methods. Perciformes have all types of reproductive systems, including gonochorism, hermaphrodite protandry, hermaphrodite protogyny, and simultaneous hermaphrodism (Avise and Mank, 2009), and these fish could serve as excellent models to study the evolution of hermaphroditism and gonochorism. As reported in the literature, hermaphroditism is widespread across teleost fish phylogeny, and no order consists solely of hermaphroditic species, suggesting that teleost fish evolved their own sexual reproduction independently in the process of adapting to environmental changes. After experiencing stress from various environmental changes, perhaps fishing pressure, larger individuals are less likely to escape capture and are forced to mature earlier; alternatively, environmental pollution from environmental endocrine disruptors may cause feminization, resulting in a population-level sex ratio imbalance. Mutations in some sex-related genes may contribute to accelerated sexual maturation, such as females always initiating meiosis before males, or may help to resist the effects of environmental endocrine disruptors to maintain a balanced sex ratio throughout the population. These may be reasons for the transition from gonochorism to hermaphroditism.

Finding a transitional species in the evolution of hermaphroditism and gonochorism in Perciformes is very important for the study of the evolution of the fish reproductive spectrum. Our previous study demonstrated that L. polyactis ' unique pattern of testis differentiation is neither typical hermaphroditism nor gonochorism (Xie et al., 2021). We speculate that this gonadal differentiation pattern inL. polyactis evolved from a gonochoristic ancestor. As mentioned above, L. polyactis has experienced severe overfishing and pollution of environmental endocrine disruptors in spawning grounds in recent decades (Jin 1996; Lin and Cheng 2004; Lu et al., 2020). Therefore, L. polyactis can be regarded as a transitional species in the process of evolution from gonochorism to hermaphroditism.

The availability of the $L$. crocea genome adds value to research on sex determination evolution. L. polyactis and $L$. croceaare the closest relatives that can interbreed (Liu et al., 2019), but they display remarkable differences in reproductive patterns and gonad development processes. $L$. crocea needs two years to reach sexual maturity, whereas $L$. polyactis is sexually mature at one year of age. Male $L$. polyactis needs to undergo a transient hermaphrodite stage, while $L$. crocea does not. Collinearity analysis of the whole genome of these two closely related species showed that almost all chromosomes of $L$. crocea and L. polyactis had high homology. The sex chromosome LG22 of L. crocea is also highly homologous with Chr. 2 of L. polyactis , except L. polyactis Chr. 2 has an inserted scaffold. In conclusion, L. polyactis is a very good model for studying the evolutionary direction between hermaphroditism and gonochorism. Whether the sexual 
reproduction type of L. polyactis will change from gonochorism to complete hermaphroditism is still an open question. The underlying molecular mechanisms warrant further study.

\section{$d m r t 1$ and its upstream gene rnf183 are candidate sex determination genes in L. polyactis}

Dmrt1 is a conserved and critical sex determination- and differentiation-related transcription factor. Dmrt1 mainly inhibits the expression of oestrogen synthesis signalling pathway factors through antagonism with Foxl2 (Forkhead box protein L2), thus inhibiting the synthesis of oestrogen to achieve testis maintenance and development ( $\mathrm{Li}$ et al., 2013). The medaka sex determination gene Dmy is a unique copy of dmrt1 on the Y chromosome (Matsuda et al., 2002; Nanda et al., 2002), and half-smooth tongue sole also uses dmrt1 (Chen S et al., 2014) as a sex-determination gene. In addition, the dmrt1gene region has been reported as a sex-specific molecular marker found inNibea albiflora , L. crocea, and Scatophagus argus(Sun et al., 2018; Lin et al., 2017; Mustapha et al., 2018).

It is interesting to note that $r n f 183$ is only $3 \mathrm{~kb}$ upstream of $d m r t 1$, and it displayed an opposite sex-biased pattern in gonad expression compared to $d m r t 1$. The RING (Really Interesting New Gene) finger protein ( $r n f$ ) family is the largest family of E3 ubiquitin ligases and contains a RING-finger domain. These members act as regulators in most cases and are widely involved in diverse aspects of growth and development, biological processes, and stress or environmental responses (Chasapis and Spyroulias, 2009; Sun et al., 2019). In mammals, RNF12 causes REX1 breakdown through dose-dependent catalysis, thereby representing an important pathway to initiate X-chromosome inactivation (Gontan et al., 2012). In humans, RING-finger protein 183 ( $R N F 183$ ) is a kidney-specific ubiquitin ligase (Kaneko et al., 2016). The abnormal expression of RNF183 is thought to be involved in several diseases, not only tumorigenesis but also inflammatory conditions such as inflammatory bowel disease (IBD). The molecular mechanism underlying the development and pathophysiology of this disease has not yet been fully elucidated (Yu et al., 2016; Okamoto et al., 2020). Although only a few studies on the role of RNFs have been reported in fish, it is clear that RNF is a regulator of downstream gene expression and molecular pathways (Yu et al., 2016). The present study revealed rapid evolution of rnf183 in fish and lineage-specific loss in some species, such as zebrafish. rnf183 is ovary-specific in L. polyactis and could drive the testis-specific expression of $d m r t 1$ through a collateral damage effect. More interestingly, rnf183 is upstream of $d m r t 1$ only in Larimichthysbut not in any other teleost species, suggesting that this innovation in gene regulation has occurred recently. The expression of rnf183shows obvious sexual dimorphism in the gonads, suggesting that this gene family plays an important role in sex determination, differentiation, and/or maintenance. The molecular mechanism requires further investigation.

Compared to the sex chromosomes in $L$. crocea, the results showed that the sex determination candidate gene $d m r t 1$ was also identified in L. polyactis . Transcriptome analysis showed that dmrt1 is the most important sex-specific gene in L. polyactis, which is expressed in the testis and not in the ovary at almost any time point. Moreover, the expression of the dmrt1 upstream gene rnf183 also showed obvious sexual dimorphism. Phylogenetic analysis showed that hermaphroditism might be related todmrt1 evolution. Therefore, we speculated that dmrt1 and $r n f 183$ are sex determination candidate genes in L. polyactis, and further research on sex-specific molecular markers is needed.

Figla, foxl2 and cyp19a1a expression patterns responsible for the male-specific transient hermaphroditic stage

Figla, foxl2 and cyp19a1a were considered to be three of the most important genes for ovarian differentiation. FIGLA is an oocyte-specific transcription factor that controls the expression of oocyte-specific zona pellucida (ZP) proteins (ZP1, ZP2, and ZP3) by binding to the E-box (CANNTG) in their promoters (Liang et al., 1997). Targeted mutagenesis of Figla in mouse resulted in ovaries that were small owing to massive depletion of oocytes and failure of primordial follicle formation (Soyal et al., 2000). In the hermaphroditic wrasse (Halichoeres poecilopterus), which displays a female-to-male sex change, figla was abundantly expressed in the ovary, and its level decreased during the sex change with oocyte degeneration. Moreover, the male-specific gene dmrt1 showed an opposite change in expression during sex reversal (Miyake et al., 2012). Overexpression of figla in XY male Nile tilapia (Oreochromis niloticus ) led to a lack of meiotic spermatocytes as well as 
spermatids (Qiu et al., 2015). In zebrafish, figla was expressed from 26 dpf, which was likely the time when gonadal differentiation occurred (Jørgensen et al., 2008). figla mutant zebrafish showed that the germ cells were clustered in cysts and underwent meiosis, forming oocytes at the prefollicular chromatin nucleolar (CN) stage (stage IA). However, the subsequent transition from cystic CN oocytes to individual follicular perinucleolar oocytes (stage IB) was blocked, resulting in an all-male phenotype in the mutant (Qin et al., 2018).

As a natural inducer of ovarian differentiation, oestrogen is considered a key factor in sex differentiation (Wang et al., 2007; Nagahama, 2000). It is well documented that foxl2 and cyp19a1a (Cytochrome P450 Family 19 Subfamily A Member 1) play a vital role in promoting ovarian development and maintaining feminization afterward in both hermaphroditic and gonochoristic teleosts (Wu and Chang, 2018; Wang et al., 2007; Guiguen et al., 2010; Li et al., 2013; Li et al., 2019). In tilapia, Cyp19a1a is expressed as early as 5 dph in XX female fish to drive the differentiation of the ovary (Xie et al., 2016). Foxl2 and Dmrt1 play antagonistic roles in sex differentiation by regulating cyp 19a1a expression and oestrogen production (Li et al., 2013). In zebrafish, oestrogen E2 or bisphenol A (BPA) primarily bindEsr2a to inhibit the expression and action of $d m r t 1$ (Song et al., 2020). Loss of function of cyp19a1a by CRISPR/Cas9 and TALENs will lead to complete masculinization in zebrafish and sexual reversion from female to male in XX tilapia, respectively (Lau et al., 2016, Zhang et al., 2017).

In L. polyactis , figla, foxl2 and cyp19a1awere also abundantly expressed in the ovary and expressed at lower levels in the testis. Interestingly, figla, foxl2 andcyp19a1a were also expressed in the testis in the male-specific transient hermaphroditic stage; however, the figla level decreased with oocyte degeneration after $80 \mathrm{dph}$, whereas foxl2and cyp19a1a expression even reached a very high level at $100 \mathrm{dph}$, but there was no significant difference between ovary and testis samples before $130 \mathrm{dph}$. These results demonstrated that figla ,foxl2, and cyp19a1a are responsible for EPO formation and the male-specific transient hermaphroditic stage.

In summary, the high-quality genome of $L$. polyactis not only provides insight into the genetic underpinnings of hermaphrodite evolution but also provides a solid foundation for sex-related gene expression dimorphism in $L$. polyactis . This is valuable information for enhancing fish aquaculture.

\section{Acknowledgments}

This work was supported by the key national and special project of blue granary science and technology innovation (grant number 2018YFD0901204); the National Natural Science Foundation of China (grant number 31902341); the Natural Science Foundation of Zhejiang Province (grant number LQ19C190002); the Science and Technology Department of Xiangshan county (grant number 2019C0001). X.W. is supported by the USDA National Institute of Food and Agriculture (Hatch project 1018100), National Science Foundation EPSCoR RII Track-4 Research Fellowship (NSF OIA 1928770), an Alabama Agricultural Experiment Station Enabling Grant, as well as a generous laboratory start-up fund from Auburn University College of Veterinary Medicine. We thank Mr. Genxing Zheng and Mr. Wantu Xu from the Xiangshan harbor aquatic seedling co. LTD, Xiangshan County, Ningbo, China for convenience of experiment materials and daily life.

\section{References}

Altschul, S. F., Madden, T. L., Schäffer, A. A., Zhang, J., Zhang, Z., Miller, W., \& Lipman, D. J. (1997). Gapped BLAST and PSI-BLAST: a new generation of protein database search programs. Nucleic acids research . 25 (17), 3389-3402. (https://doi.org/10.1093/nar/25.17.3389).

Adey, A., Kitzman, J. O., Burton, J. N., Daza, R., Kumar, A., Christiansen, L., Ronaghi, M., Amini, S., Gunderson, K. L., Steemers, F. J., \& Shendure, J. (2014). In vitro, long-range sequence information for de novo genome assembly via transposase contiguity. Genome research , 24 (12), 2041-2049. (https://doi.org/10.1101/gr.178319.114).

Attwood, T. K., \& Beck, M. E. (1994). PRINTS-a protein motif fingerprint database. Protein engineering . 7 (7), 841-848. (https://doi.org/10.1093/protein/7.7.841). 
Ashburner, M., Ball, C. A., Blake, J. A., Botstein, D., Butler, H., Cherry, J. M., Davis, A. P., Dolinski, K., Dwight, S. S., Eppig, J. T., Harris, M. A., Hill, D. P., Issel-Tarver, L., Kasarskis, A., Lewis, S., Matese, J. C., Richardson, J. E., Ringwald, M., Rubin, G. M., \& Sherlock, G. (2000). Gene ontology: tool for the unification of biology. The Gene Ontology Consortium. Nature genetics . 25 (1), 25-29. (https://doi.org/10.1038/75556).

Avise JC, Mank JE. (2009). Evolutionary perspectives on hermaphroditism in fishes. Sex Dev . 3 (2-3):15263. Review. (doi: 10.1159/000223079).

Bachtrog, D., Mank JE., Peichel, CL., Kirkpatrick, M., Otto, SP., Ashman, TL., et al. (2014). Tree of Sex Consortium Sex determination: why so many ways of doing it? PLoS Biol. 12(7):e1001899. (doi: 10.1371/journal.pbio.1001899).

Bairoch, A., \& Apweiler, R. (2000). The SWISS-PROT protein sequence database and its supplement TrEMBL in 2000. Nucleic acids research . 28 (1), 45-48. (https://doi.org/10.1093/nar/28.1.45).

Benson G. (1999). Tandem repeats finder: a program to analyze DNA sequences. Nucleic acids research , 27 (2), 573-580. (https://doi.org/10.1093/nar/27.2.573).

Birney, E., Clamp, M., \& Durbin, R. (2004). GeneWise and Genomewise.Genome research . 14 (5), 988-995. (https://doi.org/10.1101/gr.1865504).

Bru, C., Courcelle, E., Carrère, S., Beausse, Y., Dalmar, S., \& Kahn, D. (2005). The ProDom database of protein domain families: more emphasis on 3D. Nucleic acids research . 33 (Database issue), D212-D215. (https://doi.org/10.1093/nar/gki034).

Catchen, J., Amores, A., \& Bassham, S. (2020). Chromonomer: a tool set for repairing and enhancing assembled genomes through integration of genetic maps and conserved synteny. bioRxiv , 2004.934711. (doi:10.1101/2020.02.04.934711)

Chasapis, C. T., \& Spyroulias, G. A. (2009). RING finger E(3) ubiquitin ligases: structure and drug discovery. Current pharmaceutical design, 15(31), 3716-3731. (https://doi.org/10.2174/138161209789271825)

Chen, RY., Lou, B., Zhan, W., Xu, DD., Chen, L., Liu, F., et al. (2016). Broodstock cultivation and spawning induction techniques in small yellow croaker Pseudosciaena polyactis . Fish Sci.35:250-254. (DOI:10.16378/j.cnki.1003-1111.2016.03.010) (In Chinese with English abstract).

Cheng, JH., Lin, LS., Ling, JZ., Li, JS., Ding, FY. (2004). Effects of summer close season and rational utilization of redlip croaker (Larimichthys polyactis Bleeker) resource in the east China Sea region. Journal of Fishery Sciences of China . 11554-560. (http://en.cnki.com.cn/Article_en/CJFDTOTAL-ZSCK200406012.htm) (In Chinese with English abstract).

Chin, CS., Alexander, D., Marks, P. et al. (2013). Nonhybrid, finished microbial genome assemblies from long-read SMRT sequencing data.Nat Methods . 10 , 563-569. (https://doi.org/10.1038/nmeth.2474).

Darriba, D., Taboada, G. L., Doallo, R., \& Posada, D. (2012). jModelTest 2: more models, new heuristics and parallel computing. Nature methods, 9 (8), 772. (https://doi.org/10.1038/nmeth.2109).

De Bie, T., Cristianini, N., Demuth, J. P., \& Hahn, M. W. (2006). CAFE: a computational tool for the study of gene family evolution.Bioinformatics . 22 (10), 1269-1271. (https://doi.org/10.1093/bioinformatics/btlo97).

De Mitcheson, Y.S. and Liu, M. (2008). Functional hermaphroditism in teleosts. Fish and Fisheries , 9 : 1-43. (https://doi.org/10.1111/j.1467-2979.2007.00266.x).

Demuth, J. P., De Bie, T., Stajich, J. E., Cristianini, N., \& Hahn, M. W. (2006). The evolution of mammalian gene families. PloS one .1 (1), e85. (https://doi.org/10.1371/journal.pone.0000085).

Edgar R. C. (2004). MUSCLE: multiple sequence alignment with high accuracy and high throughput. Nucleic acids research .32 (5), 1792-1797. (https://doi.org/10.1093/nar/gkh340). 
Finn, R. D., Bateman, A., Clements, J., Coggill, P., Eberhardt, R. Y., Eddy, S. R., Heger, A., Hetherington, K., Holm, L., Mistry, J., Sonnhammer, E. L., Tate, J., \& Punta, M. (2014). Pfam: the protein families database. Nucleic acids research 42 (Database issue), D222-D230. (https://doi.org/10.1093/nar/gkt1223).

Gontan, C., Achame, E. M., Demmers, J., Barakat, T. S., Rentmeester, E., van IJcken, W., Grootegoed, J. A., \& Gribnau, J. (2012). RNF12 initiates X-chromosome inactivation by targeting REX1 for degradation. Nature, 485(7398), 386-390. (https://doi.org/10.1038/nature11070)

Grabherr, M. G., Haas, B. J., Yassour, M., Levin, J. Z., Thompson, D. A., Amit, I., Adiconis, X., Fan, L., Raychowdhury, R., Zeng, Q., Chen, Z., Mauceli, E., Hacohen, N., Gnirke, A., Rhind, N., di Palma, F., Birren, B. W., Nusbaum, C., Lindblad-Toh, K., Friedman, N., .. Regev, A. (2011). Full-length transcriptome assembly from RNA-Seq data without a reference genome. Nature biotechnology . 29 (7), 644-652. (https://doi.org/10.1038/nbt.1883).

Griffiths-Jones, S., Moxon, S., Marshall, M., Khanna, A., Eddy, S. R., \& Bateman, A. (2005). Rfam: annotating non-coding RNAs in complete genomes. Nucleic acids research . 33 (Database issue), D121-D124. (https://doi.org/10.1093/nar/gki081).

Guiguen Y, Fostier A, Piferrer F, Chang CF. (2010). Ovarian aromatase and estrogens: A pivotal role for gonadal sex differentiation and sex change in fish. Gen Comp Endocrinol 165:352-66. (doi: 10.1016/j.ygcen.2009.03.002).

Haas, B. J., Delcher, A. L., Mount, S. M., Wortman, J. R., Smith, R. K., Jr, Hannick, L. I., Maiti, R., Ronning, C. M., Rusch, D. B., Town, C. D., Salzberg, S. L., \& White, O. (2003). Improving the Arabidopsis genome annotation using maximal transcript alignment assemblies.Nucleic acids research . 31 (19), 56545666. (https://doi.org/10.1093/nar/gkg770).

Haas, B. J., Salzberg, S. L., Zhu, W., Pertea, M., Allen, J. E., Orvis, J., White, O., Buell, C. R., \& Wortman, J. R. (2008). Automated eukaryotic gene structure annotation using EVidenceModeler and the Program to Assemble Spliced Alignments. Genome biology ,9 (1), R7. (https://doi.org/10.1186/gb-2008-9-1-r7).

Han, KL., Le, MH., An, CM., Kim, SY., Mi, SP., Chang, YJ. (2010). Reproductive cycle of yellow croaker Larimichthys polyactis in southern waters off Korea. Fisheries Science. 76 971-980. (https://doi.org/10.1007/s12562-010-0288-5).

Hedges, S. B., Marin, J., Suleski, M., Paymer, M., \& Kumar, S. (2015). Tree of life reveals clock-like speciation and diversification.Molecular biology and evolution . 32 (4), 835-845. (https://doi.org/10.1093/molbev/msv037).

Hubley, R., Smit, A., \& Flynn, MJ. RepeatMasker at < http://www.repeatmasker.org/RepeatModeler.html $>$.

Hulo, N., Bairoch, A., Bulliard, V., Cerutti, L., De Castro, E., Langendijk-Genevaux, P. S., Pagni, M., \& Sigrist, C. J. (2006). The PROSITE database. Nucleic acids research . 34 (Database issue), D227-D230. (https://doi.org/10.1093/nar/gkj063).

Jarne, P., Auld, JR. (2006). Animals mix it up too: the distribution of self-fertilization among hermaphroditic animals. Evolution.60 1816-1824. (https://doi.org/10.1111/j.0014-3820.2006.tb00525.x).

Jones D.T., Taylor W.R., and Thornton J.M. (1992). The rapid generation of mutation data matrices from protein sequences. Computer Applications in the Biosciences 8: 275-282.

Jørgensen, A., Morthorst, J. E., Andersen, O., Rasmussen, L. J., \& Bjerregaard, P. (2008). Expression profiles for six zebrafish genes during gonadal sex differentiation. Reproductive biology and endocrinology : RBEE $E$, 6, 25. (https://doi.org/10.1186/1477-7827-6-25).

Kaneko, M., Iwase, I., Yamasaki, Y. et al. (2016). Genome-wide identification and gene expression 
profiling of ubiquitin ligases for endoplasmic reticulum protein degradation. Sci Rep 6, 30955. (https://doi.org/10.1038/srep30955)

Korf I. (2004). Gene finding in novel genomes. BMC bioinformatics . 5 , 59. (https://doi.org/10.1186/14712105-5-59).

Krzywinski, M., Schein, J., Birol, I., Connors, J., Gascoyne, R., Horsman, D., Jones, S. J., \& Marra, M. A. (2009). Circos: an information aesthetic for comparative genomics. Genome research .19 (9), 1639-1645. (https://doi.org/10.1101/gr.092759.109)

Kumar S., Stecher G., Li M., Knyaz C., and Tamura K. (2018). MEGA X: Molecular Evolutionary Genetics Analysis across computing platforms. Molecular Biology and Evolution 35:1547-1549.

Langmead, B., \& Salzberg, S. L. (2012). Fast gapped-read alignment with Bowtie 2. Nature methods . 9 (4), 357-359. (https://doi.org/10.1038/nmeth.1923).

Lau, ES., Zhang, Z., Qin, M., Ge, W. (2016). Knockout of Zebrafish Ovarian Aromatase Gene (cyp19a1a) by TALEN and CRISPR/Cas9 Leads to All-male Offspring Due to Failed Ovarian Differentiation. Sci Rep. 6 37357. (doi: 10.1038/srep37357).

Letunic, I., Doerks, T., \& Bork, P. (2012). SMART 7: recent updates to the protein domain annotation resource. Nucleic acids research $\mathbf{4 0}$ (Database issue), D302-D305. (https://doi.org/10.1093/nar/gkr931).

Li, L., Stoeckert, C. J., Jr, \& Roos, D. S. (2003). OrthoMCL: identification of ortholog groups for eukaryotic genomes. Genome research 13 (9), 2178-2189. (https://doi.org/10.1101/gr.1224503).

Li, M. H., Yang, H. H., Li, M. R., Sun, Y. L., Jiang, X. L., Xie, Q. P., Wang, T. R., Shi, H. J., Sun, L. N., Zhou, L. Y., \& Wang, D. S. (2013). Antagonistic roles of Dmrt1 and Foxl2 in sex differentiation via estrogen production in tilapia as demonstrated by TALENs.Endocrinology , 154(12), 4814-4825. (https://doi.org/10.1210/en.2013-1451).

Li M, Sun L, Wang D. Roles of estrogens in fish sexual plasticity and sex differentiation. Gen Comp Endocrinol (2019) 277:9-16. (doi: 10.1016/ j.ygcen.2018.11.015).

Li, H., \& Durbin, R. (2009). Fast and accurate short read alignment with Burrows-Wheeler transform. Bioinformatics , 25 (14), 1754-1760. (https://doi.org/10.1093/bioinformatics/btp324).

Li R, Fan W, Tian G, et al. (2010). The sequence and de novo assembly of the giant panda genome. Nature . 463(7279):311. (doi:10.1038/nature08696).

Li, B., \& Dewey, C. N. (2011). RSEM: accurate transcript quantification from RNA-Seq data with or without a reference genome. BMC bioinformatics . 12, 323. (https://doi.org/10.1186/1471-2105-12-323).

Liang, L., Soyal, S. M., \& Dean, J. (1997). FIGalpha, a germ cell-specific transcription factor involved in the coordinate expression of the zona pellucida genes. Development (Cambridge, England), 124(24), 4939-4947. (https://dev.biologists.org/content/124/24/4939.long).

Lin, A., Xiao, S., Xu, S., Ye, K., Lin, X., Sun, S., \& Wang, Z. (2017). Identification of a male-specific DNA marker in the large yellow croaker (Larimichthys crocea ). Aquaculture , 480 , 116-122. (https://doi.org/10.1016/j.aquaculture.2017.08.009).

Lin, LS., Cheng, JH. (2004). An analysis of the current situation of fishery biology of small yellow croaker in the east china sea. Journal of Ocean University of China. 34(4) : 565-70. (DOI:10.16441/j.cnki.hdxb.2004.04.009) (In Chinese with English abstract)

Liu B, Shi Y, Yuan J, et al. (2013). Estimation of genomic characteristics by analyzing k-mer frequency in de novo genome projects.Quantitative Biology . 35 (s 1-3):62-67. (https://arxiv.org/abs/1308.2012). 
Liu F, Zhan W, Xie Q, Chen H, Lou B, Xu W. (2020). A first genetic linage map construction and QTL mapping for growth traits inLarimichthys polyactis . Sci Rep. 15 ;10(1):11621. (doi: 10.1038/s41598-02068592-0).

Liu, F., Liu, Y., Chu, T. Lou B., Zhan W., Chen R.Y. (2019). Interspecific hybridization and genetic characterization ofLarimichthys polyactis () and L. crocea ().Aquacult Int 27 , 663-674. (https://doi.org/10.1007/s10499-019-00353-x).

Liu, M., Wang, YY., Shan, XJ., Kang, B., Ding, SX. (2016). Primary male development of two sequentially hermaphroditic groupers, Epinephelus akaara and Epinephelus awoara (Perciformes: Epinephelidae). J Fish Biol. 88 (4):1598-613. (https://doi.org/10.1111/jfb.12936).

Lu, J., Zhang, C., Wu, J., Zhang, Y., \& Lin, Y. (2020). Seasonal distribution, risks, and sources of endocrine disrupting chemicals in coastal waters: Will these emerging contaminants pose potential risks in marine environment at continental-scale?. Chemosphere , 247, 125907. (https://doi.org/10.1016/j.chemosphere.2020.125907)

Lowe, T. M., \& Eddy, S. R. (1997). tRNAscan-SE: a program for improved detection of transfer RNA genes in genomic sequence. Nucleic acids research . 25 (5), 955-964. (https://doi.org/10.1093/nar/25.5.955).

Matsuda, M., Nagahama, Y., Shinomiya, A., Sato, T., Matsuda, C., Kobayashi, T., Morrey, C. E., Shibata, N., Asakawa, S., Shimizu, N., Hori, H., Hamaguchi, S., \& Sakaizumi, M. (2002). DMY is a Yspecific DM-domain gene required for male development in the medaka fish.Nature , 417 (6888), 559-563. (https://doi.org/10.1038/nature751).

Majoros, W. H., Pertea, M., \& Salzberg, S. L. (2004). TigrScan and GlimmerHMM: two open source ab initio eukaryotic gene-finders.Bioinformatics . $\quad 20$ (16), 2878-2879. (https://doi.org/10.1093/bioinformatics/bth315).

Mi, H., Lazareva-Ulitsky, B., Loo, R., Kejariwal, A., Vandergriff, J., Rabkin, S., Guo, N., Muruganujan, A., Doremieux, O., Campbell, M. J., Kitano, H., \& Thomas, P. D. (2005). The PANTHER database of protein families, subfamilies, functions and pathways. Nucleic acids research . 33 (Database issue), D284-D288. (https://doi.org/10.1093/nar/gki078).

Mistry, J., Finn, R. D., Eddy, S. R., Bateman, A., \& Punta, M. (2013). Challenges in homology search: HMMER3 and convergent evolution of coiled-coil regions. Nucleic acids research . 41 (12), e121. (https://doi.org/10.1093/nar/gkt263).

Miyake, Y., Sakai, Y., \& Kuniyoshi, H. (2012). Molecular cloning and expression profile of sex-specific genes, Figla and Dmrt1, in the protogynous hermaphroditic fish, Halichoeres poecilopterus .Zoological science, 29(10), 690-701. (https://doi.org/10.2108/zsj.29.690).

Mulder, N., \& Apweiler, R. (2007). InterPro and InterProScan: tools for protein sequence classification and comparison. Methods in molecular biology . 396 , 59-70. (https://doi.org/10.1007/978-1-59745-515-2_5).

Mustapha, U. F., Jiang, D. N., Liang, Z. H., Gu, H. T., Yang, W., Chen, H. P., .. \& Li, G. L. (2018). Male-specific Dmrt1 is a candidate sex determination gene in spotted scat (Scatophagus argus ). Aquaculture , 495 , 351-358. (https://doi.org/10.1016/j.aquaculture.2018.06.009).

Myers G. (2014). Efficient Local Alignment Discovery amongst Noisy Long Reads. In: Brown D., Morgenstern B. (eds) Algorithms in Bioinformatics. WABI 2014. Lecture Notes in Computer Science, vol 8701. Springer, Berlin, Heidelberg. (https://doi.org/10.1007/978-3-662-44753-6_5).

Nagahama Y. Gonadal steroid hormones: major regulators of gonadal sex differ- entiation and gametogenesis in fish. In: B Norberg, OS Kjesbu, GL Taranger, E Andersson, SO Stefansson, editors. Proceedings of the $6^{\text {th }}$ International Symposium on Reproductive Physiology of Fish. Bergen, Norway: Institute of Marine Research and University of Bergen (2000). p. 211-22. Available at: http://www.vliz.be/en/imis?refid=4219 
Nanda, I., Kondo, M., Hornung, U., Asakawa, S., Winkler, C., Shimizu, A., Shan, Z., Haaf, T., Shimizu, N., Shima, A., Schmid, M., \& Schartl, M. (2002). A duplicated copy of DMRT1 in the sex-determining region of the Y chromosome of the medaka, Oryzias latipes .Proceedings of the National Academy of Sciences of the United States of America, 99 (18), 11778-11783. (https://doi.org/10.1073/pnas.182314699)

Nawrocki, E. P., Kolbe, D. L., \& Eddy, S. R. (2009). Infernal 1.0: inference of RNA alignments. Bioinformatics . 25 (10), 1335-1337. (https://doi.org/10.1093/bioinformatics/btp157).

Nei M. and Kumar S. (2000). Molecular Evolution and Phylogenetics.Oxford University Press, New York.

Ogata, H., Goto, S., Sato, K., Fujibuchi, W., Bono, H., \& Kanehisa, M. (1999). KEGG: Kyoto Encyclopedia of Genes and Genomes. Nucleic acids research . 27 (1), 29-34. (https://doi.org/10.1093/nar/27.1.29).

Okamoto, T., Imaizumi, K., \& Kaneko, M. (2020). The Role of Tissue-Specific Ubiquitin Ligases, RNF183, RNF186, RNF182 and RNF152, in Disease and Biological Function. International journal of molecular sciences, 21(11), 3921. (https://doi.org/10.3390/ijms2111392)

Orban, L., Sreenivasan, R., Olsson, PE. (2009). Long and winding roads: testis differentiation in zebrafish. Mol Cell Endocrinol.312 (1-2):35-41. (https://doi.org/10.1016/j.mce.2009.04.014).

Park IS, Gil HW. (2019). Karyotypic Analysis of Chicken Grunt, Parapristipoma trilimeatum, Small Yellow Croaker, Larimichthys polyactis, and Brown Croaker, Miichthys miiuy. Dev Reprod.23 (1):73-78. (doi: 10.12717/DR.2019.23.1.073).

Parra, G., Blanco, E., \& Guigo, R. (2000). GeneID in Drosophila.Genome research . 10 (4), 511-515. (https://doi.org/10.1101/gr.10.4.511).

Parra, G., Bradnam, K., \& Korf, I. (2007). CEGMA: a pipeline to accurately annotate core genes in eukaryotic genomes.Bioinformatics . 23 (9), 1061-1067. (https://doi.org/10.1093/bioinformatics/btm071).

Pendleton, M., Sebra, R., Pang, A. W., Ummat, A., Franzen, O., Rausch, T., Stutz, A. M., Stedman, W., Anantharaman, T., Hastie, A., Dai, H., Fritz, M. H., Cao, H., Cohain, A., Deikus, G., Durrett, R. E., Blanchard, S. C., Altman, R., Chin, C. S., Guo, Y., .. Bashir, A. (2015). Assembly and diploid architecture of an individual human genome via single-molecule technologies. Nature methods . 12 (8), 780-786. (https://doi.org/10.1038/nmeth.3454).

Price, A. L., Jones, N. C., \& Pevzner, P. A. (2005). De novo identification of repeat families in large genomes.Bioinformatics . 21 Suppl 1, i351-i358. (https://doi.org/10.1093/bioinformatics/bti1018).

Qin, M., Zhang, Z., Song, W., Wong, Q. W., Chen, W., Shirgaonkar, N., \& Ge, W. (2018). Roles of Figla/figla in Juvenile Ovary Development and Follicle Formation During Zebrafish Gonadogenesis. Endocrinology , 159(11), 3699-3722. (https://doi.org/10.1210/en.2018-00648).

Qiu, Y., Sun, S., Charkraborty, T., Wu, L., Sun, L., Wei, J., Nagahama, Y., Wang, D., \& Zhou, L. (2015). Figla Favors Ovarian Differentiation by Antagonizing Spermatogenesis in a Teleosts, Nile Tilapia (Oreochromis niloticus ). PloS one, 10(4), e0123900. (https://doi.org/10.1371/journal.pone.0123900).

Renner, S.S. and Ricklefs, R.E. (1995). Dioecy and its correlates in the flowering plants. American Journal of Botany. 82 596-606. (doi:10.1002/j.1537-2197.1995.tb11504.x).

Robinson, M. D., McCarthy, D. J., \& Smyth, G. K. (2010). edgeR: a Bioconductor package for differential expression analysis of digital gene expression data. Bioinformatics . 26 (1), 139-140. (https://doi.org/10.1093/bioinformatics/btp616).

Robinson, M. D., \& Oshlack, A. (2010). A scaling normalization method for differential expression analysis of RNA-seq data. Genome biology . 11 (3), R25. (https://doi.org/10.1186/gb-2010-11-3-r25).

Salamov, A. A., \& Solovyev, V. V. (2000). Ab initio gene finding in Drosophila genomic DNA. Genome research 10 (4), 516-522. (https://doi.org/10.1101/gr.10.4.516). 
Song WY, Lu HJ, Wu K, Zhang ZW, Lau ESW, Ge W. (2020). Genetic Evidence for Estrogenicity of Bisphenol A in Zebrafish Gonadal Differentiation and Its Signalling Mechanism. J Hazard Mater . 386:121886. (doi: 10.1016/j.jhazmat.2019.121886).

Soyal, S. M., Amleh, A., \& Dean, J. (2000). FIGalpha, a germ cell-specific transcription factor required for ovarian follicle formation. Development , 127(21), 4645-4654. (https://dev.biologists.org/content/127/21/4645.short).

Schaefer H, Renner SS. (2010). A three-genome phylogeny of Momordica (Cucurbitaceae) suggests seven returns from dioecy to monoecy and recent long-distance dispersal to Asia. Mol Phylogenet Evol.54(2):55360. (doi: 10.1016/j.ympev.2009.08.006).

Simao, F. A., Waterhouse, R. M., Ioannidis, P., Kriventseva, E. V., \& Zdobnov, E. M. (2015). BUSCO: assessing genome assembly and annotation completeness with single-copy orthologs. Bioinformatics .31 (19), 3210-3212. (https://doi.org/10.1093/bioinformatics/btv351).

Stamatakis A. (2006). RAxML-VI-HPC: maximum likelihood-based phylogenetic analyses with thousands of taxa and mixed models.Bioinformatics . 22 (21), 2688-2690. (https://doi.org/10.1093/bioinformatics/btl446).

Stamatakis, A., Hoover, P., \& Rougemont, J. (2008). A rapid bootstrap algorithm for the RAxML Web servers. Systematic biology .57 (5), 758-771. (https://doi.org/10.1080/10635150802429642).

Stamatakis A. (2014). RAxML version 8: a tool for phylogenetic analysis and postanalysis of large phylogenies. Bioinformatics (Oxford, England), 30(9), 1312-1313. (https://doi.org/10.1093/bioinformatics/btu033).

Stanke, M., \& Waack, S. (2003). Gene prediction with a hidden Markov model and a new intron submodel. Bioinformatics . 19Suppl 2, ii215-ii225. (https://doi.org/10.1093/bioinformatics/btg1080).

Stanke, M., Schoffmann, O., Morgenstern, B., \& Waack, S. (2006). Gene prediction in eukaryotes with a generalized hidden Markov model that uses hints from external sources. BMC bioinformatics . 7 , 62 . (https://doi.org/10.1186/1471-2105-7-62).

Stecher G., Tamura K., and Kumar S. (2020). Molecular Evolutionary Genetics Analysis (MEGA) for macOS. Molecular Biology and Evolution (https://doi.org/10.1093/molbev/msz312).

Sun, J., Sun, Y., Ahmed, R. I., Ren, A., \& Xie, A. M. (2019). Research Progress on Plant RING-Finger Proteins. Genes, 10(12), 973. (https://doi.org/10.3390/genes10120973)

Sun, S., Li, W., Xiao, S., Lin, A., Han, Z., Cai, M., \& Wang, Z. (2018). Genetic sex identification and the potential sex determination system in the yellow drum (Nibea albiflora). Aquaculture .492, 253-258. (https://doi.org/10.1016/j.aquaculture.2018.03.042).

Sun, Y. B., Zhou, W. P., Liu, H. Q., Irwin, D. M., Shen, Y. Y., \& Zhang, Y. P. (2013). Genome-wide scans for candidate genes involved in the aquatic adaptation of dolphins. Genome biology and evolution .5 (1), 130-139. (https://doi.org/10.1093/gbe/evs123).

Tang, H., Wang, X., Bowers, J. E., Ming, R., Alam, M., \& Paterson, A. H. (2008). Unraveling ancient hexaploidy through multiply-aligned angiosperm gene maps. Genome Research . 18 , 1944-1954. (https://doi.org/10.1101/gr.080978.108).

Tang, H., Bowers, J. E., Wang, X., Ming, R., Alam, M., \& Paterson, A. H. (2008). Synteny and collinearity in plant genomes. Science (New York, N.Y.), 320(5875), 486-488. (https://doi.org/10.1126/science.1153917).

Takahashi, H. (1977). Juvenile hermaphroditism in the zebrafish, Brachydanio rerio . Bull Fac Fish Hokkaido Univ .28:57-65. (http://hdl.handle.net/2115/23605) 
Tarailo-Graovac, M., \& Chen, N. (2009). Using RepeatMasker to identify repetitive elements in genomic sequences. Current protocols in bioinformatics. Chapter 4. (https://doi.org/10.1002/0471250953.bi0410s25).

Trapnell, C., Pachter, L., \& Salzberg, S. L. (2009). TopHat: discovering splice junctions with RNA-Seq. Bioinformatics .25 (9), 1105-1111. (https://doi.org/10.1093/bioinformatics/btp120).

Trapnell, C., Williams, B. A., Pertea, G., Mortazavi, A., Kwan, G., van Baren, M. J., Salzberg, S. L., Wold, B. J., \& Pachter, L. (2010). Transcript assembly and quantification by RNA-Seq reveals unannotated transcripts and isoform switching during cell differentiation. Nature biotechnology . 28 (5), 511-515. (https://doi.org/10.1038/nbt.1621).

Tree of Sex consortium: Ashman, TL., Bachtrog, D., Blackmon, H., Goldberg, EE., Hahn, MW., Kirkpatrick, M., Kitano, J., Mank, JE., Mayrose, I., Ming, R., Otto, SP., Peichel, CL., Pennell, MW., Perrin, N., Ross, L., Valenzuela, N., Vamosi, JC. (2014). Tree of Sex: A database of sexual systems. Sci Data . 1 :140015. (doi: 10.1038/sdata.2014.15).

Walker, B.J., Abeel, T., Shea, T., Priest, M., Abouelliel, A., Sakthikumar S, et al. (2014). Pilon: An Integrated Tool for Comprehensive Microbial Variant Detection and Genome Assembly Improvement. PLoS ONE. 9 (11): e112963. (https://doi.org/10.1371/journal.pone.0112963).

Wang DS, Kobayashi T, Zhou LY, Paul-Prasanth B, Ijiri S, Sakai F, et al. Foxl2 upregulates aromatase gene transcription in a female-specific manner by binding to the promoter as well as interacting with ad4 binding protein/steroidogenic factor 1. Mol Endocrinol (2007) 21(3):712-25. (doi: 10.1210/me.2006-0248).

Wang, Z., Pascual-Anaya, J., Zadissa, A., Li, W., Niimura, Y., Huang, Z., Li, C., White, S., Xiong, Z., Fang, D., Wang, B., Ming, Y., Chen, Y., Zheng, Y., Kuraku, S., Pignatelli, M., Herrero, J., Beal, K., Nozawa, M., Li, Q., ... Irie, N. (2013). The draft genomes of soft-shell turtle and green sea turtle yield insights into the development and evolution of the turtle-specific body plan. Nature genetics , 45 (6), 701-706. (https://doi.org/10.1038/ng.2615).

Wu GC, Chang CF. Primary males guide the femaleness through the regulation of testicular Dmrt1 and ovarian Cyp19a1a in protandrous black porgy. Gen Comp Endocrinol (2018) 261:198-202. (doi: 10.1016/j.ygcen.2017.01.033)

Xie QP, He X, Sui YN, Chen LL, Sun LN, Wang DS. (2016). Haploinsufficiency of SF-1 Causes Female to Male Sex Reversal in Nile Tilapia, Oreochromis niloticus . Endocrinology157(6):2500-14. (doi: 10.1210/en.2015-2049).

Xie QP, Li BB, Zhan W, Liu F, Tan P, Wang X, Lou B. (2021). A Transient Hermaphroditic Stage in Early Male Gonadal Development in Little Yellow Croaker, Larimichthys polyactis . Front Endocrinol(Lausanne). 11 :542942. (doi: 10.3389/fendo.2020.542942).

$\mathrm{Xu}$, Z., \& Wang, H. (2007). LTR_FINDER: an efficient tool for the prediction of fulllength LTR retrotransposons. Nucleic acids research. $\mathbf{3 5}$ (Web Server issue), W265-W268. (https://doi.org/10.1093/nar/gkm286).

Yang Z. (2007). PAML 4: phylogenetic analysis by maximum likelihood.Molecular biology and evolution, 24 (8), 1586-1591. (https://doi.org/10.1093/molbev/msm088).

Yang, J., Chen, X., Bai, J., Fang, D., Qiu, Y., Jiang, W., Yuan, H., Bian, C., Lu, J., He, S., Pan, X., Zhang, Y., Wang, X., You, X., Wang, Y., Sun, Y., Mao, D., Liu, Y., Fan, G., Zhang, H., .. Shi, Q. (2016). The Sinocyclocheilus cavefish genome provides insights into cave adaptation. BMC biology , $14,1$. (https://doi.org/10.1186/s12915-015-0223-4).

You, XR., Cai, MY., Jiang, YH., Wang, ZY. (2012). Histological observation on gonadal sex differentiation in large yellow croaker (Larimichthys crocea ). Journal of Fisheries of China. 36 (7): 1057-1064. (http://en.cnki.com.cn/Article_en/CJFDTotal-SCKX201207010.htm) (In Chinese with English abstract) 
Yu, Q., Zhang, S., Chao, K., Feng, R., Wang, H., Li, M., Chen, B., He, Y., Zeng, Z., \& Chen, M. (2016). E3 Ubiquitin ligase RNF183 Is a Novel Regulator in Inflammatory Bowel Disease. Journal of Crohn's \& colitis, 10(6), 713-725. (https://doi.org/10.1093/ecco-jcc/jjw02)

Zhang, J., Nielsen, R., \& Yang, Z. (2005). Evaluation of an improved branch-site likelihood method for detecting positive selection at the molecular level. Molecular biology and evolution .22 (12), 2472-2479. (https://doi.org/10.1093/molbev/msi237).

Zhang, G., Cowled, C., Shi, Z., Huang, Z., Bishop-Lilly, K. A., Fang, X., Wynne, J. W., Xiong, Z., Baker, M. L., Zhao, W., Tachedjian, M., Zhu, Y., Zhou, P., Jiang, X., Ng, J., Yang, L., Wu, L., Xiao, J., Feng, Y., Chen, Y., ... Wang, J. (2013). Comparative analysis of bat genomes provides insight into the evolution of flight and immunity.Science. 339 (6118), 456-460. (https://doi.org/10.1126/science.1230835).

Zhang, BD., Xue, DX., Wang, J., Li, YL., Liu, BJ., Liu, JX. (2016). Development and preliminary evaluation of a genome-wide single nucleotide polymorphisms resource generated by RAD-seq for the small yellow croaker (Larimichthys polyactis ). Mol Ecol Resour.16 755-768. (https://doi.org/10.1111/1755-0998.12476).

Zhang X, Li M, Ma H, Liu X, Shi H, Li M, et al. (2017). Mutation of foxl2 or cyp19a1a Results in Female to Male Sex Reversal in XX Nile Tilapia. Endocrinology. 158(8):2634-47. (doi: 10.1210/en.2017-00127).

\section{Data Accessibility}

The raw genome and RNA sequencing data have been deposited in the Sequence Read Archive (SRA) database under Bioproject number PRJNA705840. The final chromosome assembly of L. polyactis has been submitted to NCBI (National Center for Biotechnology Information,https://www.ncbi.nlm.nih.gov) Assembly under accession number JAFMOB000000000. The version described in this paper is version JAFMOB000000000.

\section{Conflict of Interest}

The authors declare that they have no competing interests.

\section{Author Contributions}

Conceived and designed the experiments: Q.X., X.W. and B.L.; collected sample: Q.X., X. H, W.Z., F.L.; performed the experiments: Q.X., X. H. and J. S.; performed the data analyses: Q.X, J. S., B.N., Y. X., M. L., Q. L., P. X. and X.W; wrote the paper: Q.X. X.W. and B.L. All authors contributed to manuscript revision, read and approved the submitted version.

\section{Figure legends}

\section{Figure 1. Characteristics of the Larimichthys polyactis genome assembly.}

(A) A picture showing female and male little yellow croaker, L polyactis . (B) Comparison of the gene sets obtained using different prediction methods (de novo, homology-based, RNA-seq-based). Over $84.3 \%$ of 25233 genes were supported by at least one prediction method. (C) Divergence distribution of classified families of TEs. Red indicates DNA transposons (DNAs), dark yellow indicates long interspersed nuclear elements (LINEs), green indicates long terminal repeats (LTRs), light blue indicates short interspersed nuclear elements (SINEs), and purple indicates unknown TEs.(D) The Venn diagram shows common and unique gene families among the four species. The four species include Larimichthys crocea (Lcr), Gasterosteus aculeatus (Gac), Danio rerio(Dre) and Larimichthys polyactis (Lpo). (E) Protein orthology comparison among the genomes of eighteen species. The eighteen species include Larimichthys polyactis (Lpo), Larimichthys crocea (Lcr), Oreochromis niloticus (Oni), Gasterosteus aculeatus (Gac), Oryzias latipes (Ola), Takifugu rubripes(Tru), Cynoglossus semilaevis (Cse), Danio rerio (Dre),Homo sapiens (Hsa), Mus musculus (Mmu), Gallus gallus (Gga), Branchiostoma floridae (Bf), Lepisosteus oculatus (Loc), Callorhinchus milii (Cmi), Gadus morhua(Gmo), Ciona intestinalis (Cin), Anolis carolinensis (Aca) and Xenopus tropicalis (Xtr). Single-copy orthologues are marked with pink, multiple-copy orthologues are marked with yellow, unique genes are marked with dark yellow, and other orthologues are marked with green. 
Figure 2. Phylogenetic and gene expansion and contraction analysis of Larimichthys polyactis.

Phylogenetic tree of 18 vertebrate genomes constructed using 592 single-copy orthologous genes. The numbers on the branches indicate the estimated diverge times in millions of years ago, and red circles indicate the calibration time from fossil Homo sapiens (Hsa), Mus musculus (Mmu), Gallus gallus (Gga), Anolis carolinensis (Aca), Xenopus tropicalis (Xtr). Danio rerio(Dre), Gadus morhua (Gmo), Cynoglossus semilaevis (Cse), Gasterosteus aculeatus (Gac), Larimichthys polyactis(Lpo), Larimichthys crocea (Lcr), Takifugu rubripes (Tru), Oryzias latipes (Ola), Oreochromis niloticus (Oni),Lepisosteus oculatus (Loc), Callorhinchus milii (Cmi), Ciona intestinalis (Cin) and Branchiostoma floridae (Bfl).

Figure 3. Genome comparisons between Larimichthys croceaand Larimichthys polyactis.

Twenty-four chromosomes of L. polyactis show a one-to-one relationship with 24 Larimichthys crocea chromosomes. Linkage groups 1-24 on the left of the circle represent Larimichthys crocea chromosomes, and chromosomes 1-24 on the right represent $L$. polyactis chromosomes.

Figure 4. Sex chromosome comparisons between Larimichthys crocea and Larimichthys polyactis.

(A) Genome collinearity analysis of sex chromosomes inLarimichthys crocea and L. polyactis . The lines between the two horizontal lines link the alignment blocks. Plus and minus represent the direction of gene transcription. The positions of genes related to sex and reproduction are marked on chromosomes. (B) Enlarged view of the region between piwi2 and rnf122 on sex chromosomes. (C) Phylogenetic tree of rnf family members on sex chromosomes of Larimichthys crocea and L. polyactis. The evolutionary history was inferred by using the maximum likelihood method and general time reversible model. The tree is drawn to scale, with branch lengths measured as the number of substitutions per site. Evolutionary analyses were conducted in MEGA X.

Figure 5. Transcriptome correlation and heat map analysis.

Transcriptome analysis of three mixed pools of whole-fish samples of $L$. polyactis at 7, 10, 20, and $30 \mathrm{dph}$, where each pool had six fishes; from three mixed pools of indistinguishable gonads at $40 \mathrm{dph}$, where each pool had six gonads; and from thirteen pairs of ovary and testis mixed pools at 50, 60, 70, 80, 100, 130, $180,240,270,340,360,400$, and $720 \mathrm{dph}$, where each pool had three samples. The results showed that the correlation of gonads of different sexes at different periods had high quality and good correlation clustering. The positions of sex determination, differentiation or maintenance genes, including cyp19a1a, figla, rnf183 , foxl2 ,rnf225, gsdf , amh and dmrt1, are marked in the picture.

Figure 6. dmrt1 gene is involved in the hermaphrodite intersex stage in L. polyactis.

(A) Phylogenetic tree of dmrt1 in hermaphroditic and gonochorical vertebrates. The evolutionary history was inferred by using the maximum likelihood method and general time reversible model. Evolutionary analyses were conducted in MEGA X. For the different sexual reproduction patterns, please refer to the legend in the figure.(B) Syntenic analyses of $d m r t 1$ and its adjacent genes in humans (Homo sapiens ), mice (Mus musculus ), lizards (Anolis carolinensis ), frogs (Xenopus laevis ), tilapia (Oreochromis niloticus ), zebrafish (Danio rerio), fugu (Takifugu rubripes), seabass (Lates calcarifer), and large (Larimichthys crocea) and little yellow croaker (Larimichthys polyactis ). The NCBI gene browser (https://www.ncbi.nlm.nih.gov) was used to make this figure. Gene symbols are described according to the browser. L. polyactis' gene symbols are based on the genome annotation. (C) Gene structure of $d m r t 1$ in different teleosts. The yellow rectangle represents exon 1, the light green rectangle represents exon 2, the dark green rectangle represents exon 3 , the blue rectangle represents exon 4 and the red rectangle represents exon 5 . The numbers below the black line represent the number of bases. (D) The gene structure of $d m r t 1$ and its upstream gene rnf183 . rnf183 has two exons and is $3197 \mathrm{bp}$ away from $d m r t 1$; the brown rectangle represents rnf183 transcripts. (E) Tissue distribution and expression profiles of dmrt1 in different developmental stages of the ovary and testis. Five indistinguishable sex stages and thirteen pairs of ovaries and testes from 7, 10, 20,30,40,50,60, 70, 80, 100, 130, 180 240, 270, 340, 360, 400 and $720 \mathrm{dph}$ (days post hatching) were sequenced using Illumina 2000 HiSeq 
technology in the present study. A normalized measure of RPKM (reads per kilobase per million reads) was used to normalize the expression profiles of $d m r t 1$. Different uppercase and lowercase letters above the error bars indicate significant differences in females or males at different time points, respectively, at $\mathrm{P}<0.05$, as determined by one-way ANOVA followed by Duncan's post hoc test. * and ** represent a significant difference at $\mathrm{P}<0.05$ and $\mathrm{P}<0.01$ between females and males, respectively, by independent t-test.

Figure 7. Phylogenetic analysis and expression profile of $r$ f183 and its paralogues rnf223 andrnf225 in L. polyactis

(A) Phylogenetic tree of rnf183, rnf223, and rnf225 in vertebrates. The evolutionary history was inferred by using the maximum likelihood method and general time reversible model. Evolutionary analyses were conducted in MEGA X. (B)Syntenic analyses of rnf183 and its adjacent genes in humans (Homo sapiens ), mice (Mus musculus ), lizards (Anolis carolinensis), frogs (Xenopus laevis ), tilapia (Oreochromis niloticus), zebrafish (Danio rerio), seabass (Lates calcarifer), and large (Larimichthys crocea) and little yellow croaker (Larimichthys polyactis ). The NCBI gene browser (https://www.ncbi.nlm.nih.gov) was used to make this figure. Gene symbols are described according to the browser. L. polyactis' gene symbols are based on the genome annotation.(C) Tissue distribution and expression profiles ofrnf183, rnf223 and rnf225 and in different developmental stages of the ovary and testis. The results are presented as the means +- SD from a sample of three individuals for each data point. Different lowercase and uppercase letters above the error bar indicate significant differences in females and males at different time points, respectively, at $\mathrm{P}<0.05$, as determined by one-way ANOVA followed by Duncan's post hoc test. * and ** represent a significant difference at $\mathrm{P}<0.05$ and $\mathrm{P}<0.01$ between females and males at the same time point, respectively, by independent t-test.

Figure 8. Sex-related gene expression profile in L. polyactis gonads.

Expression profiles of amh, gsdf, cyp19a1a, figla and foxl2 based on tissue distributions and different developmental stages of the ovary and testis. The results are presented as the means +- SD from a sample of three individuals for each data point. Different lowercase and uppercase letters above the error bars indicate significant differences in females and males at different time points, respectively, at $\mathrm{P}<0.05$, as determined by one-way ANOVA followed by Duncan's post hoc test. ${ }^{*}$ and ${ }^{* *}$ represent a significant difference at $\mathrm{P}<$ 0.05 and $\mathrm{P}<0.01$ between females and males at the same time point, respectively, by independent t-test.

Tables

Table 1 The statistics of sequencing data for L. polyactis genome Assembly

\begin{tabular}{lllll}
\hline Sequencing types & Insert size & Total data $(\mathbf{G})$ & Read length (bp) & Sequence coverage $(\mathbf{X})$ \\
\hline Pacbio reads & $20 \mathrm{~Kb}$ & 48.12 & - & 68.74 \\
Illumina & $350 \mathrm{bp}$ & 128.37 & 150 & 183.39 \\
10X Genomics & - & 137.28 & 150 & 196.11 \\
bionano & - & 117 & - & 167.6 \\
Total & - & 430.77 & - & 615.84 \\
\hline
\end{tabular}

Table 2 Summary of L. polyactis genome assembly statistics

\begin{tabular}{ll}
\hline Total length & $706,148,513 \mathrm{bp}$ \\
\hline Number of scaffolds & 715 \\
Number of contigs & 3,134 \\
Longest scaffold & $20,429,779 \mathrm{bp}$ \\
Contig N50 length & $1,208,045 \mathrm{bp}$ \\
Scaffold N50 length & $4,519,237 \mathrm{bp}$ \\
Scaffold N90 length & $389,991 \mathrm{bp}$
\end{tabular}




\begin{tabular}{ll}
\hline Total length & $706,148,513 \mathrm{bp}$ \\
\hline Genome characteristics & Genome characteristics \\
Percent assembled & $102 \%$ \\
GC content & $41.49 \%$ \\
Contents of repetitive elements & $22.28 \%$ \\
Content of transposable elements & $21.26 \%$ \\
Heterozygous SNP number and rate (SNPs per kb) & $3,895,963(0.575 \%)$ \\
Predicted protein-coding gene number & 25,233 \\
Predicted non-coding RNA gene number & 5,671 \\
Quantity of scaffolds anchored on chromosomes & 309 \\
Proportion of the genome is anchored to chromosomes & $88.55 \%$ \\
Length of scaffolds anchored on chromosomes & $625,267,019 \mathrm{bp}$ \\
\hline
\end{tabular}

GC Guanine/Cytosine

\section{Figures}

Figure 1.

A

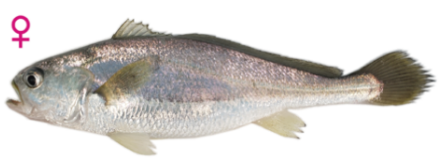

ऽ

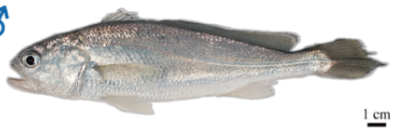

B

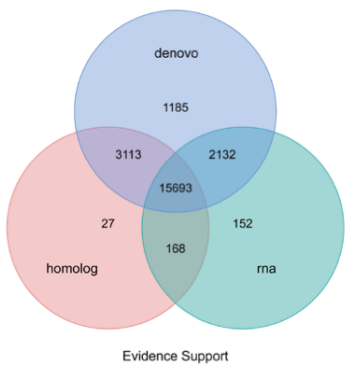

C

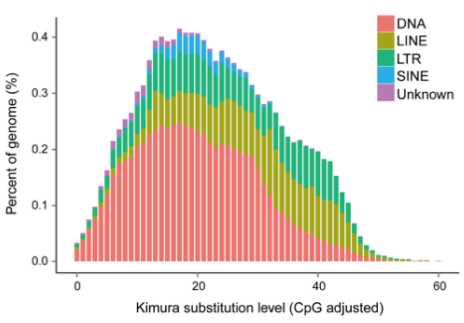

D

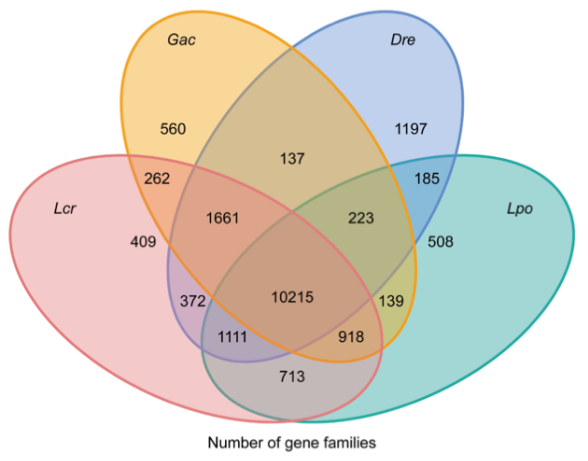

$\mathbf{E}$

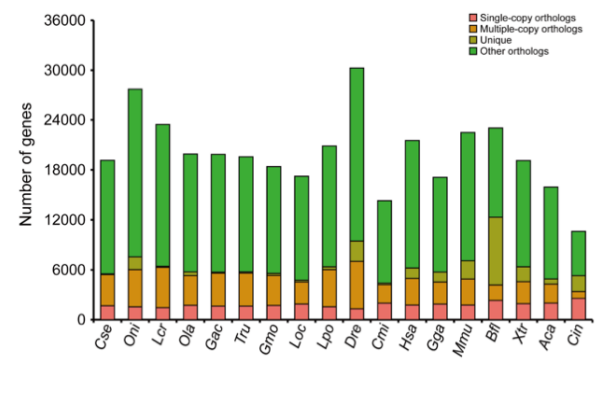

Figure 2. 


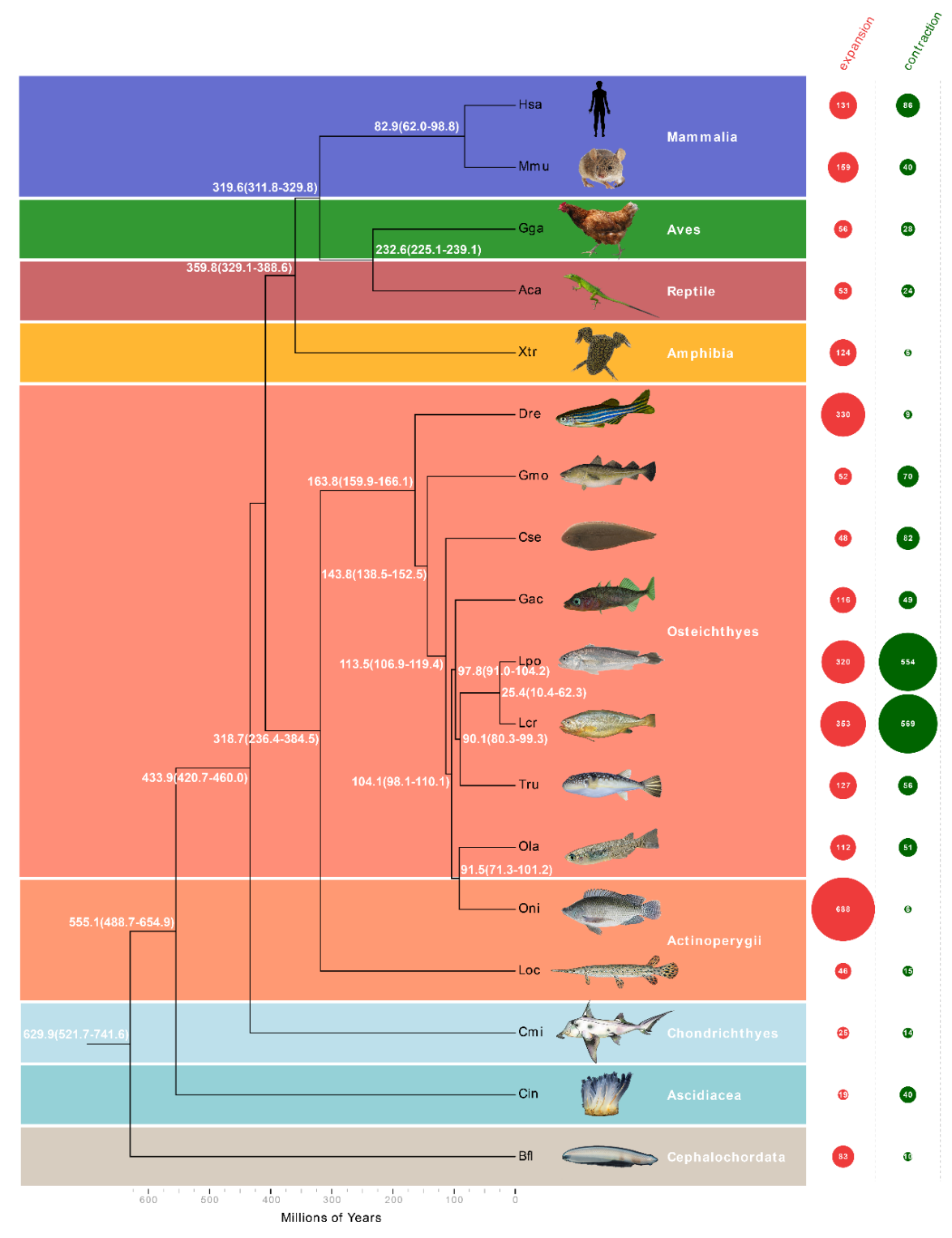

Figure 3. 


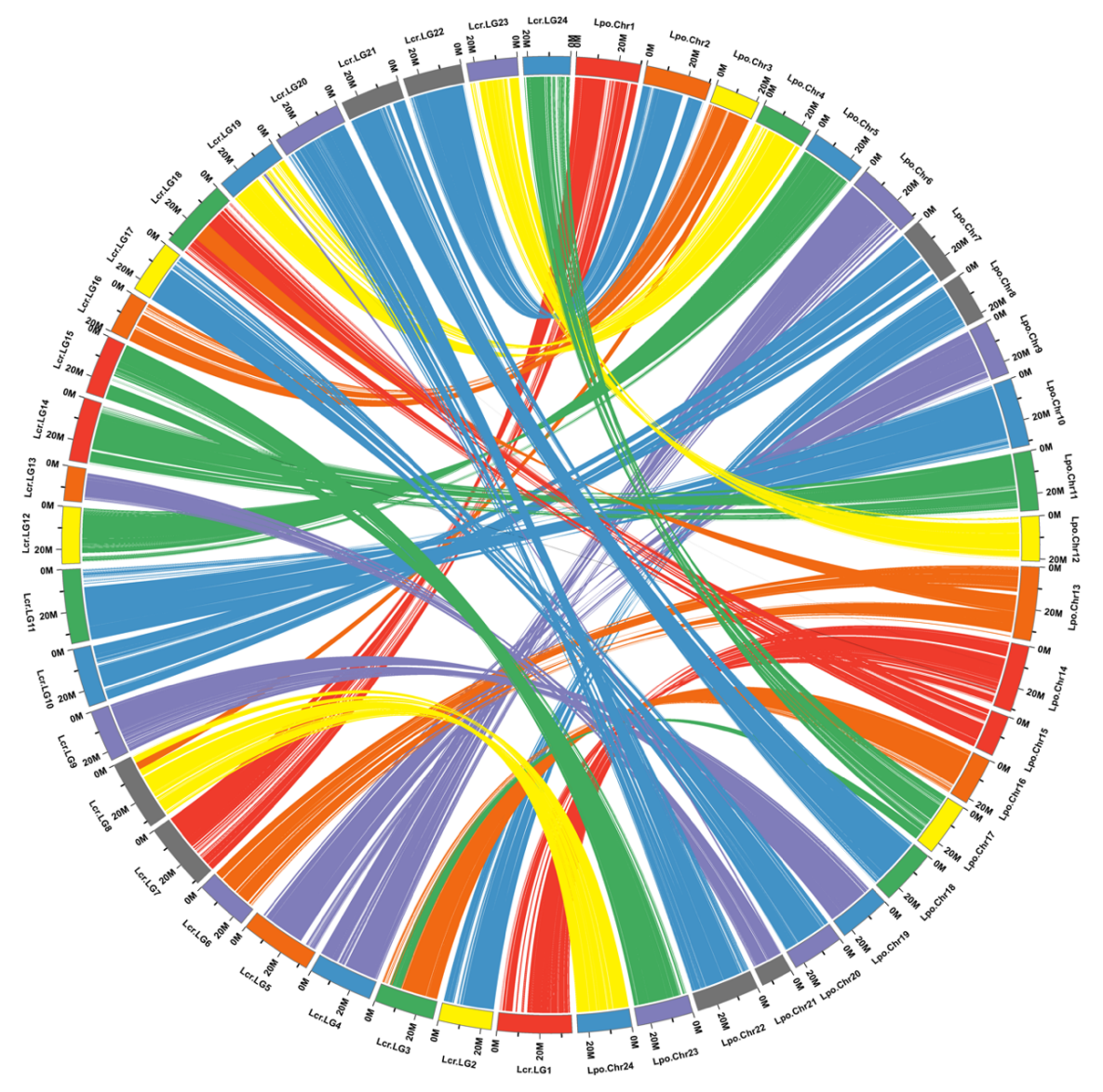

Figure 4. 


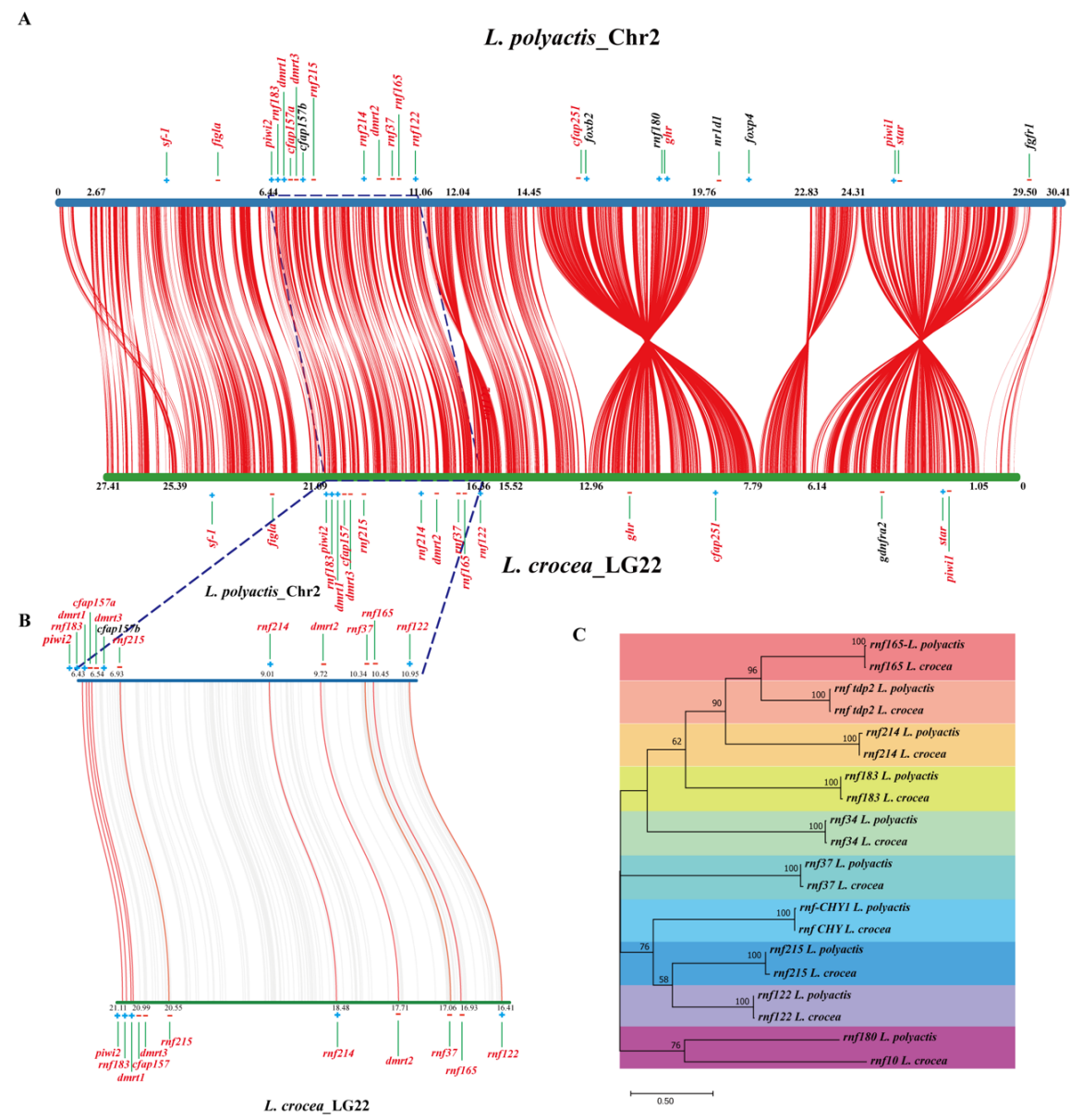

Figure 5. 


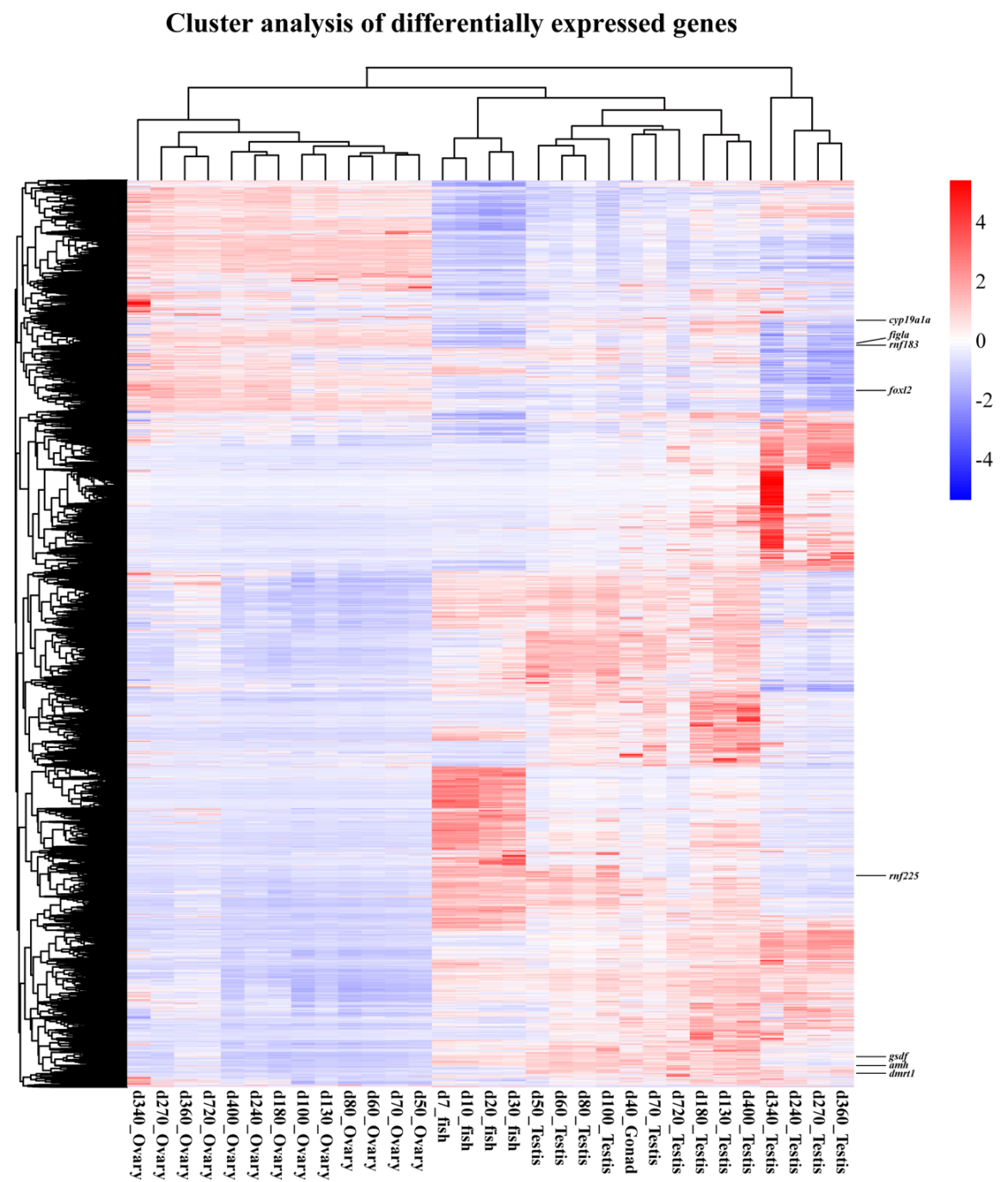

Figure 6. 


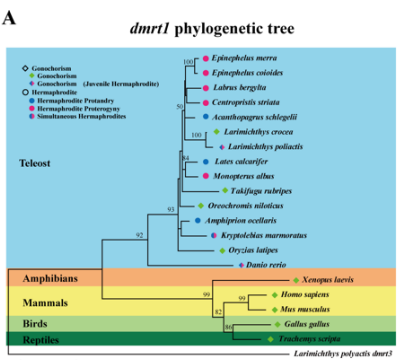

B Home sapains Mus masculus Andis cerrolinnisis

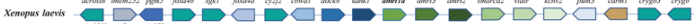
Damio rerio

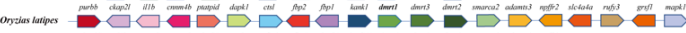

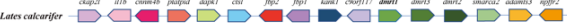

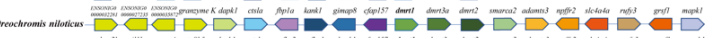

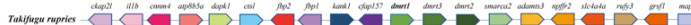

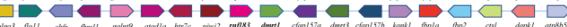

D

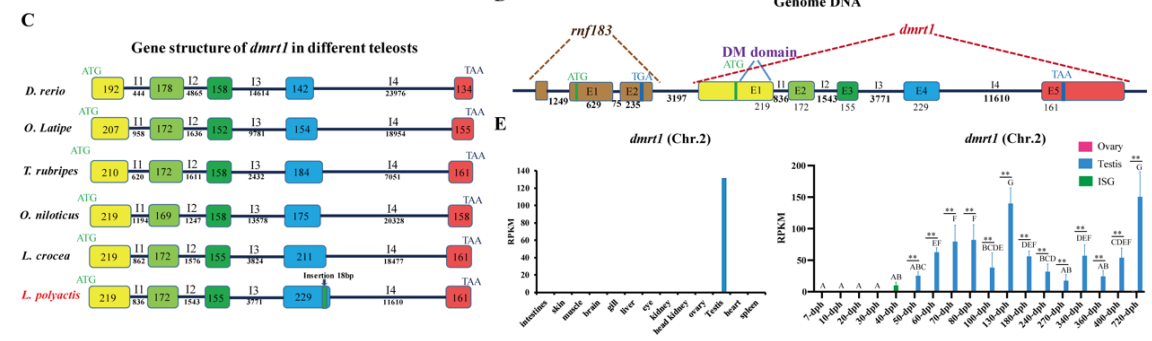

Figure 7.

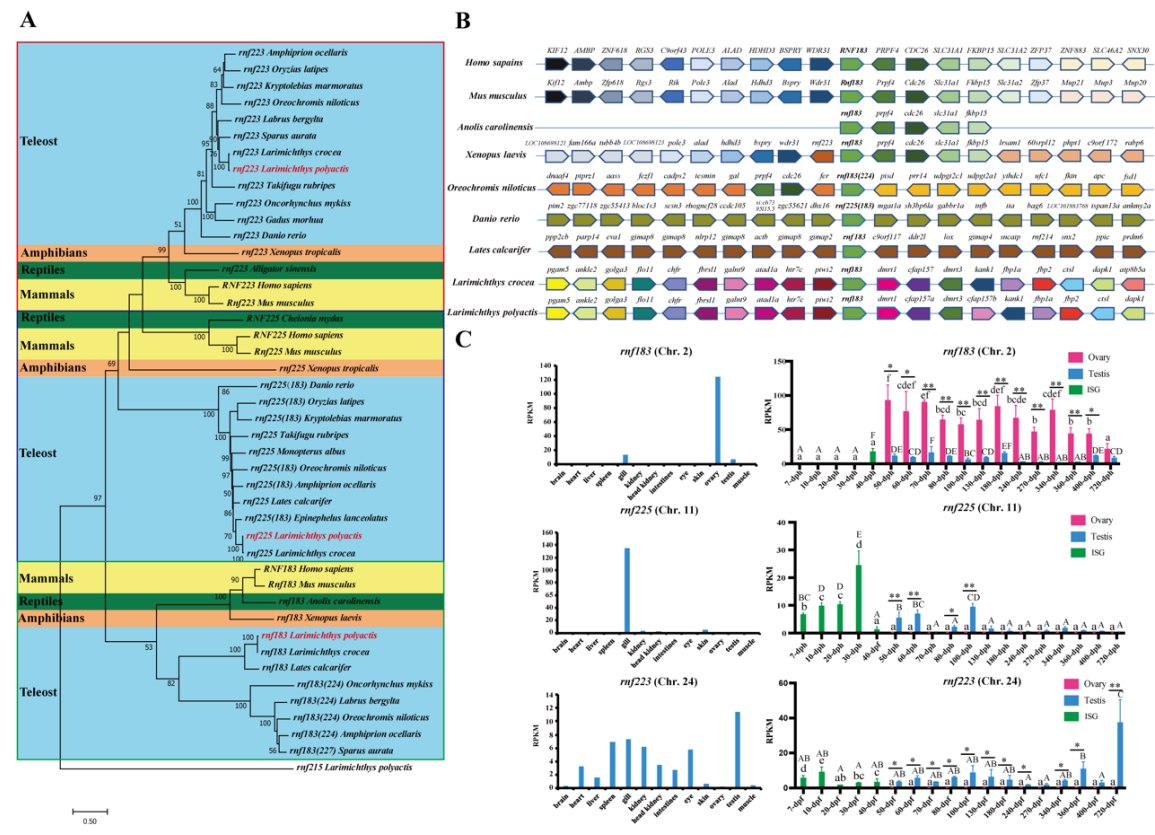

Figure 8. 

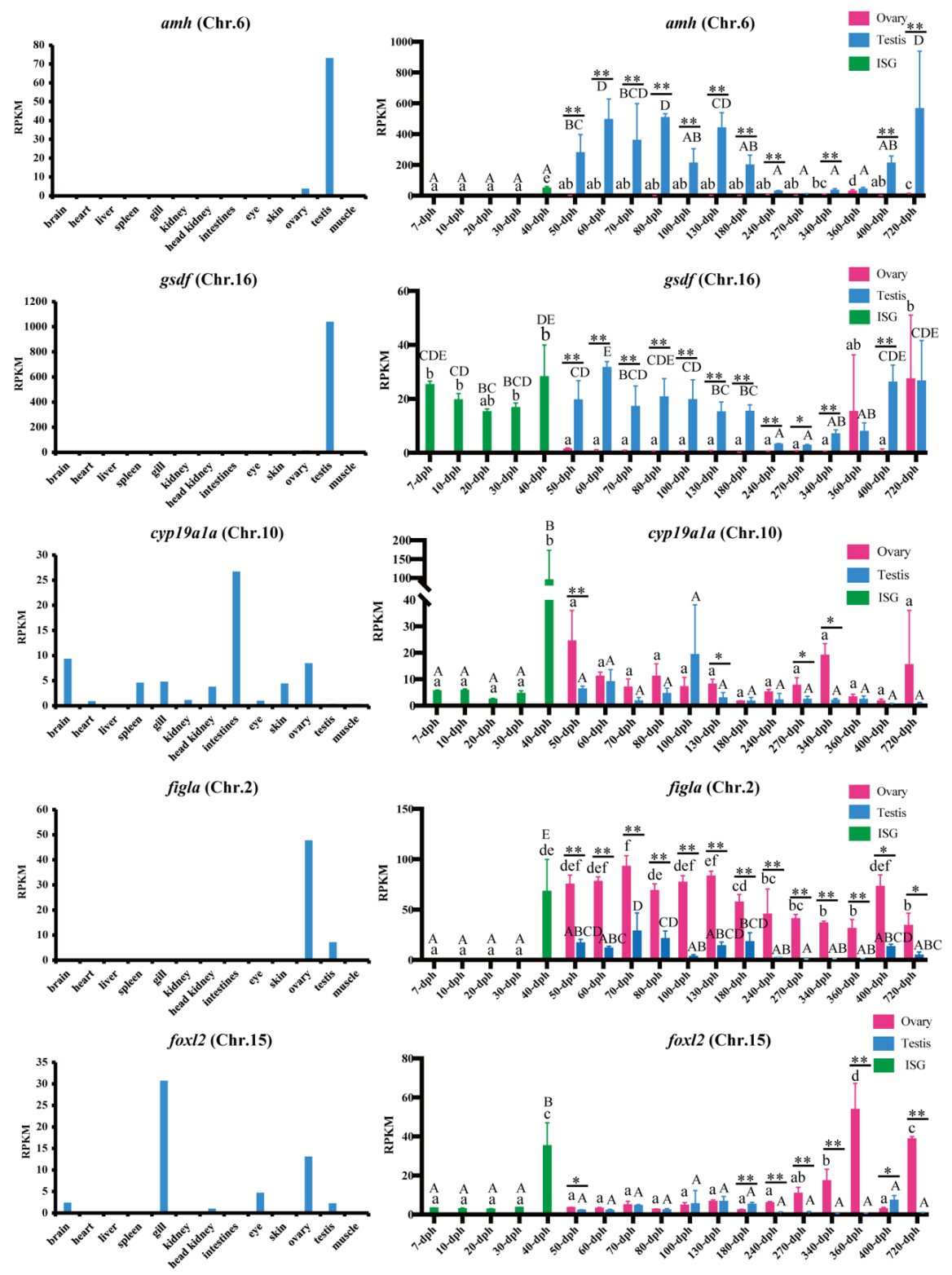